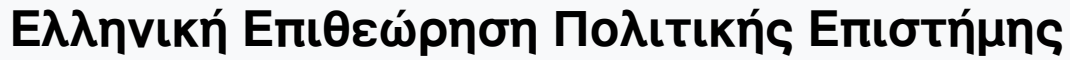

Tó 17 (2001)

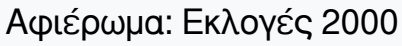

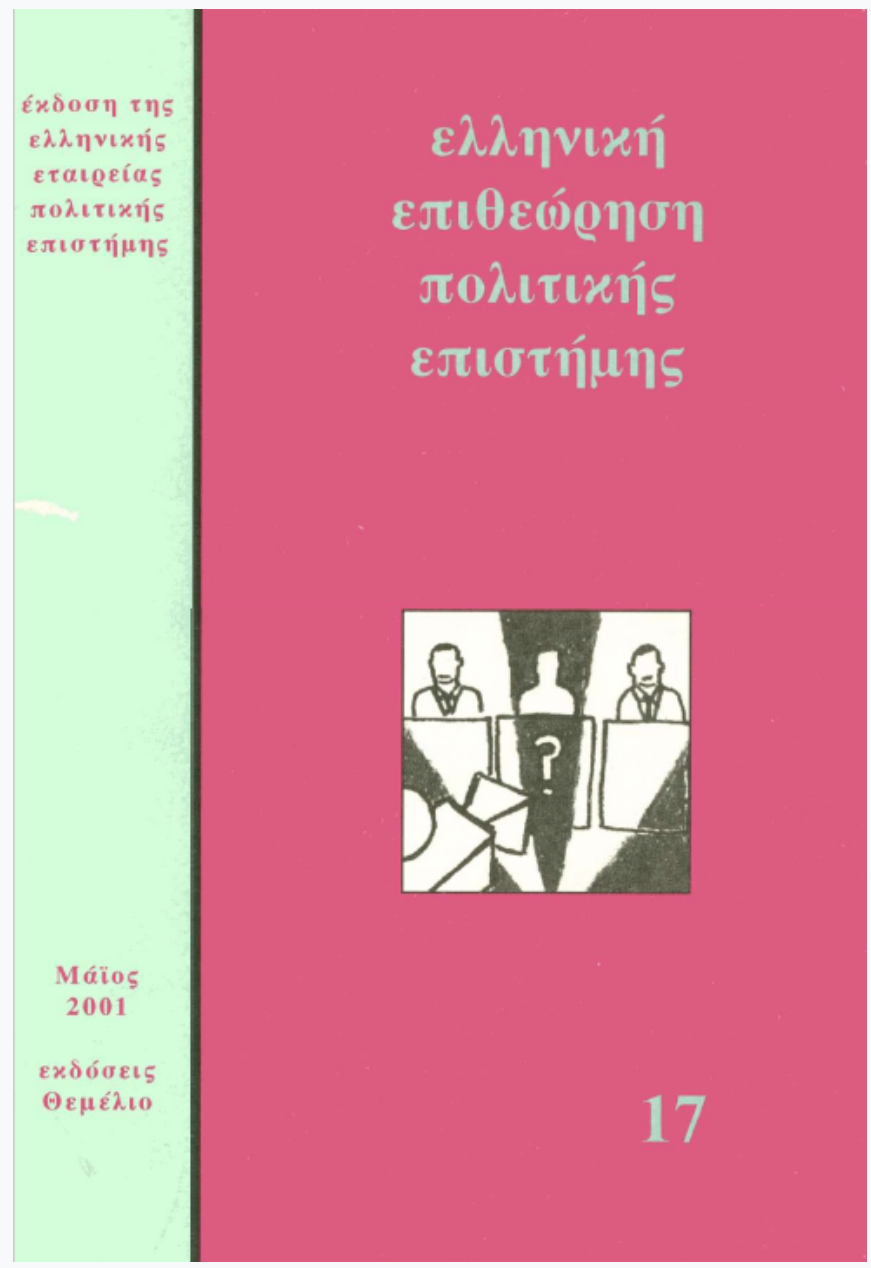

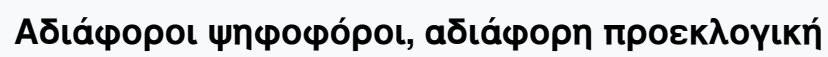

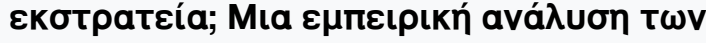
ßou

\section{Mavíva Какєпа́кп}

doi: $10.12681 /$ hpsa.15194

\section{Copyright @ 2017, Mavíva Kaкєпákn}

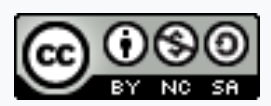

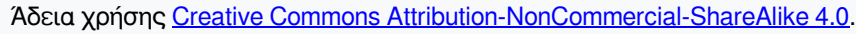

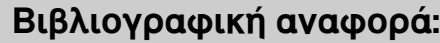

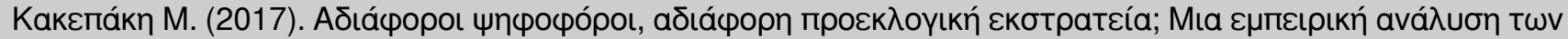

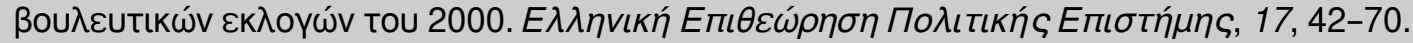

https://doi.org/10.12681/hpsa.15194 


\title{
MANINA KAKEIIAKH*
}

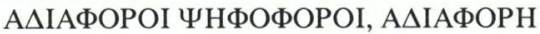

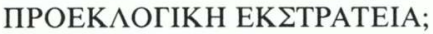

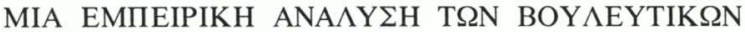

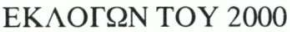

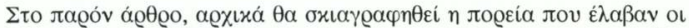

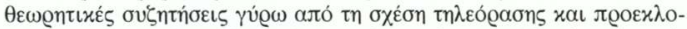

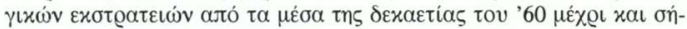

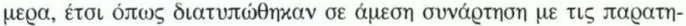

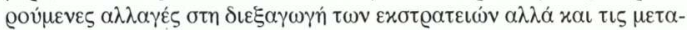

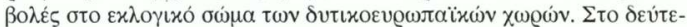

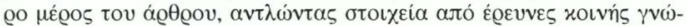

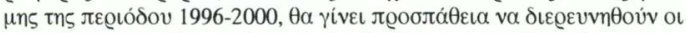

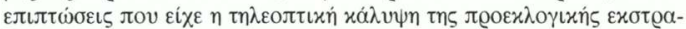

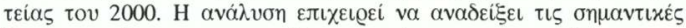

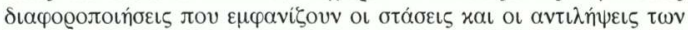

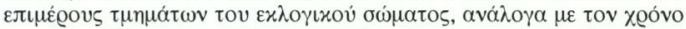

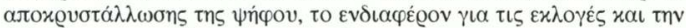

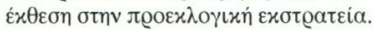

\section{1. $\mathrm{EI \Sigma AГ \Omega \Gamma H}$}

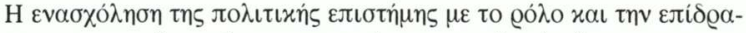

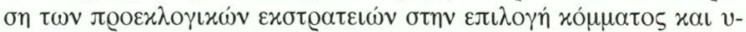

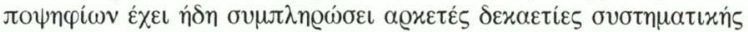

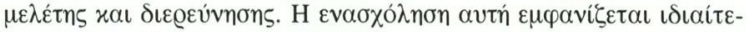

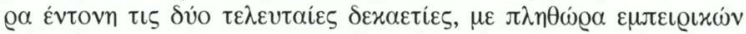

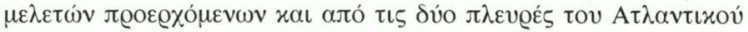

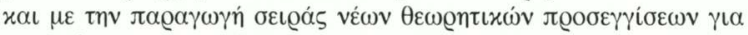

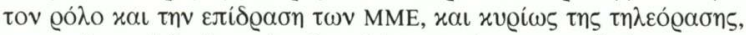

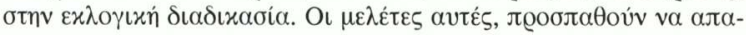

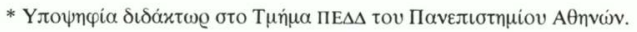




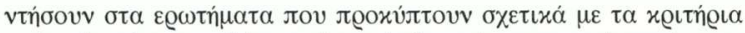

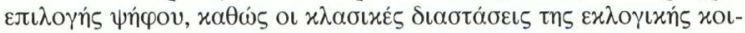

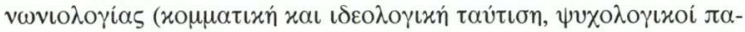

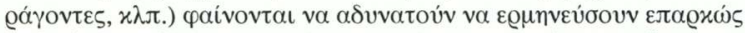

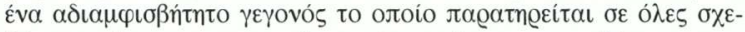

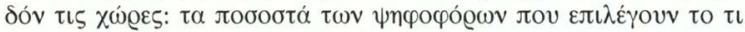

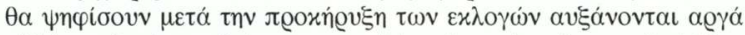

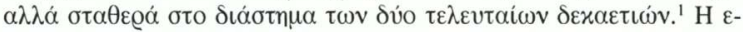

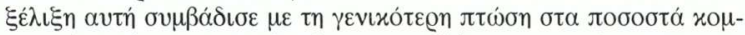

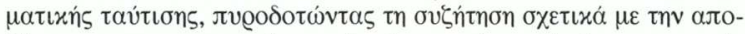

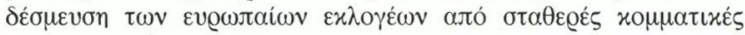

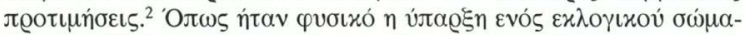

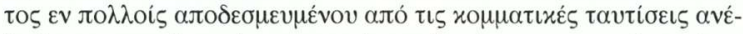

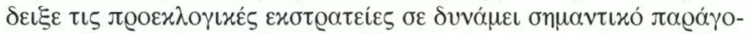

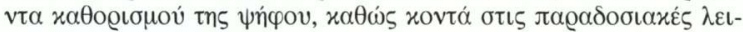

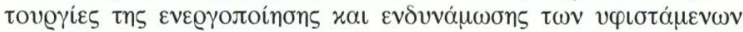

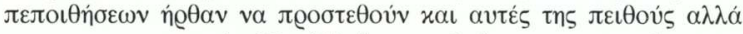

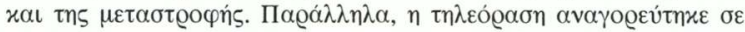

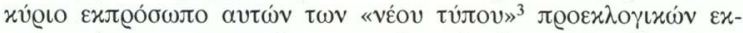

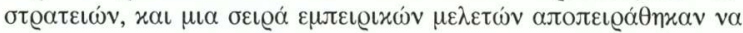

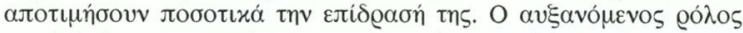

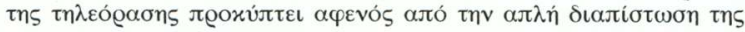

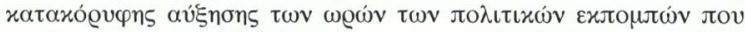

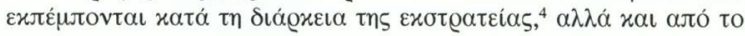

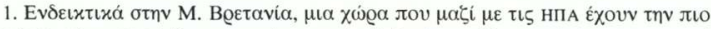

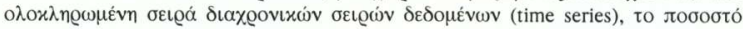

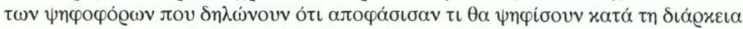

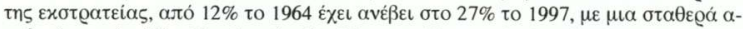

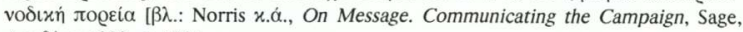

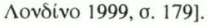

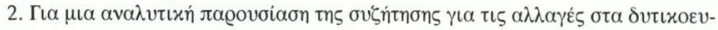

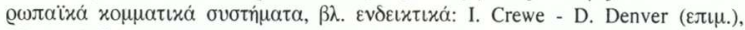
Electoral Change in Western Democracies. Patterns and Sources of Instability, Croom

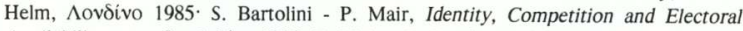
Availability, CuP, Cambridge 1990• P. Mair - G. Smith), Understanding Party System

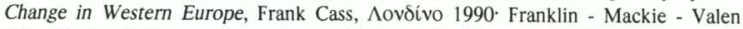
x.á., Electoral Change: Responses to Evolving Social and Attitudinal Structures in Western Europe, CuP, Cambridge 1992 J.E. Lane - S. Ersson, Politics and Society in

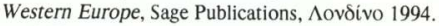

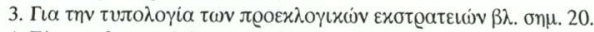

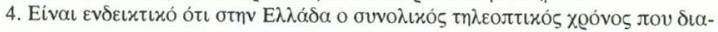




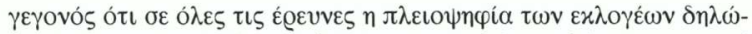

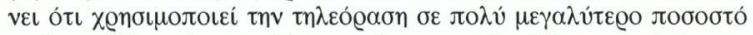

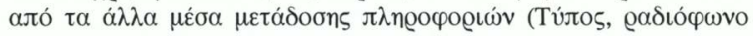

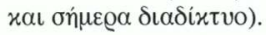

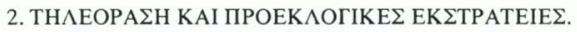

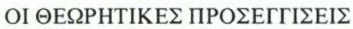

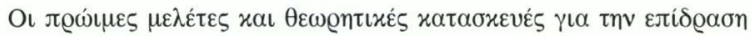

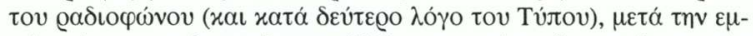

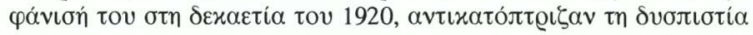

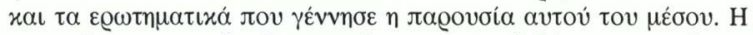

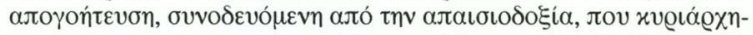

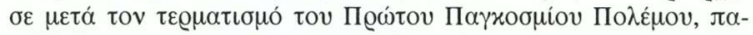

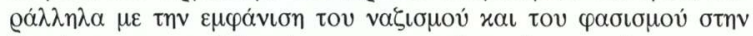

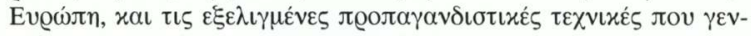

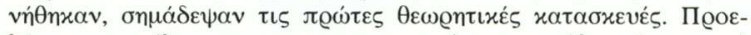

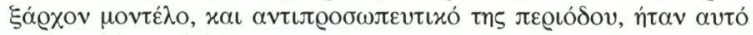

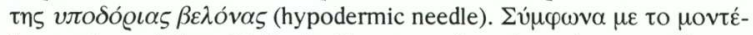

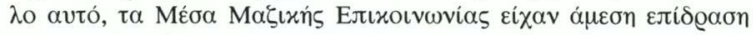

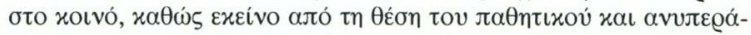

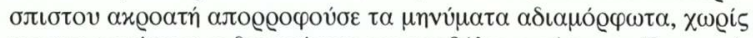

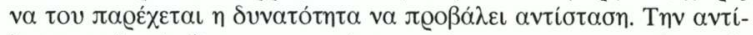

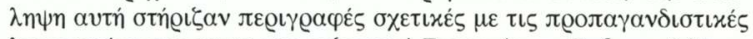

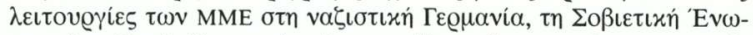

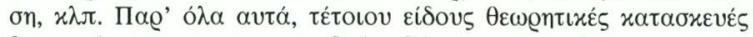

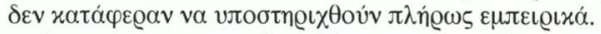

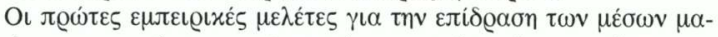

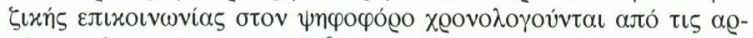

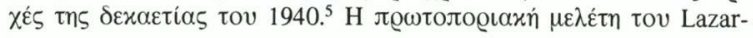

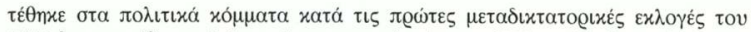

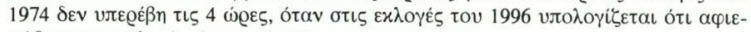

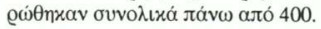

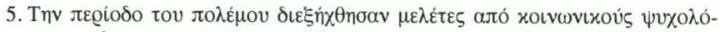

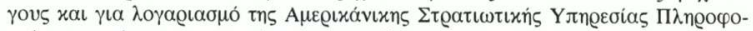

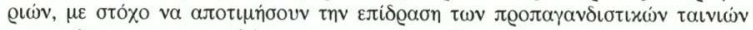

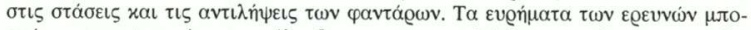

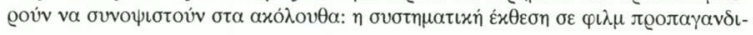




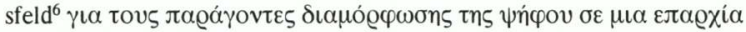

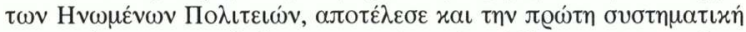

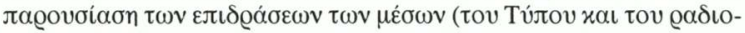

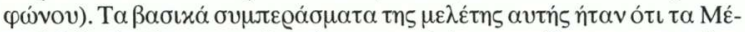

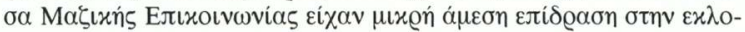

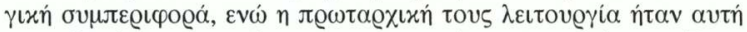

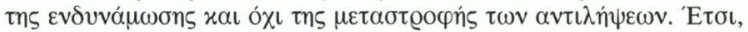

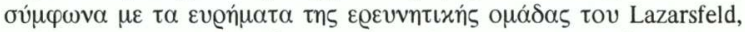
ol

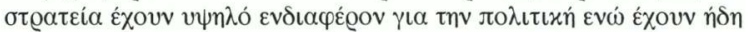

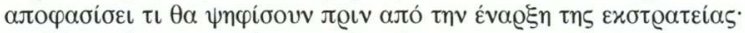

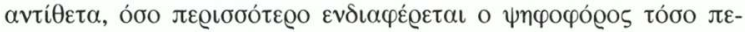

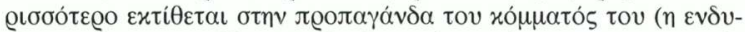

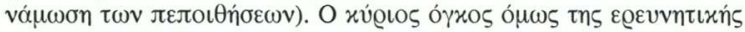

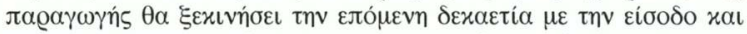

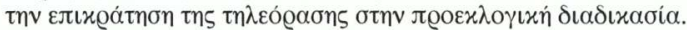

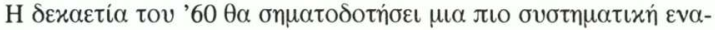

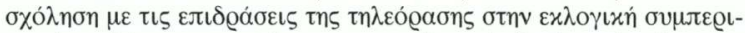

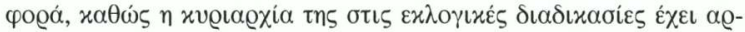

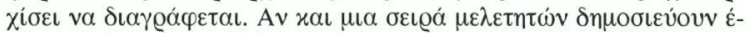

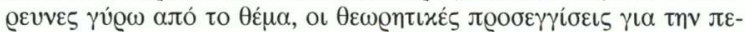

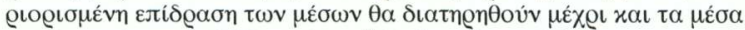

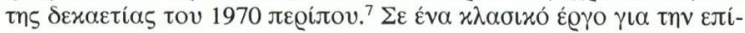

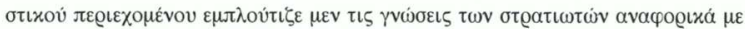

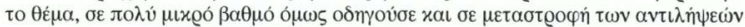

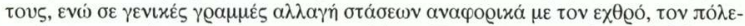

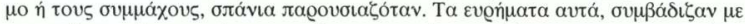

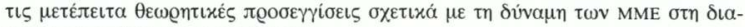

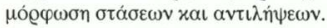

6. P. Lazarsfeld, The People's Choice. How the voter makes up his mind in a presi-

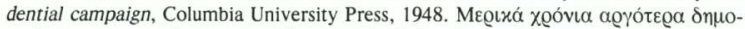

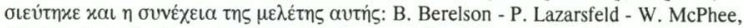
Voting: A study of opinion formation in a presidential campaign, Chicago University

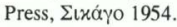

7. O Joseph Klapper oto $\beta$ « $\beta \lambda$ io tov The Effects of Mass Communication, Free

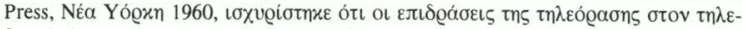

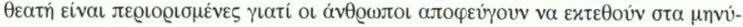

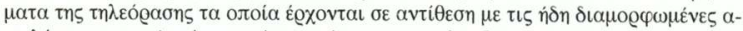

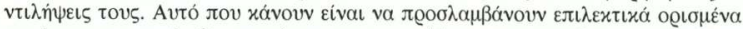

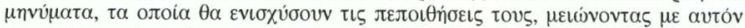

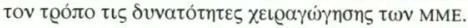




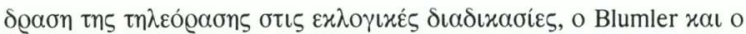

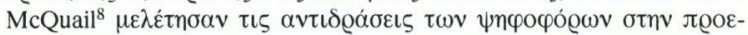

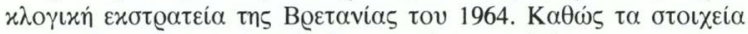

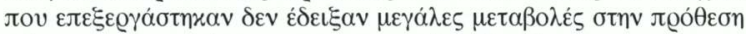

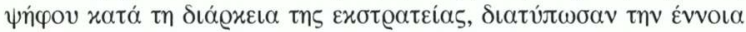

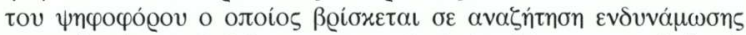

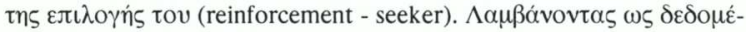

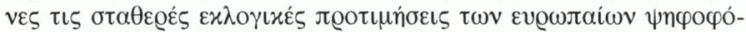

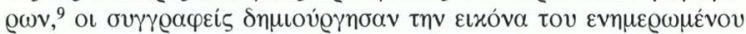

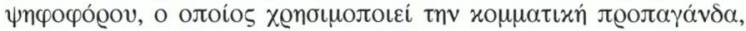

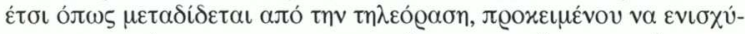

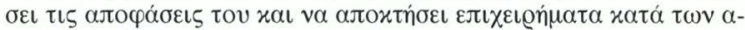

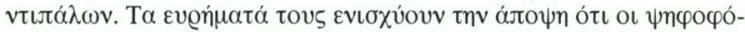

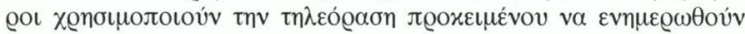

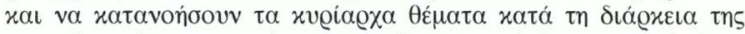

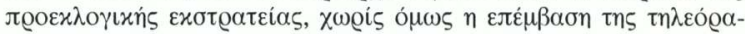

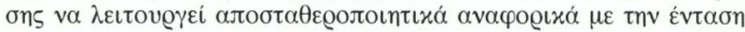

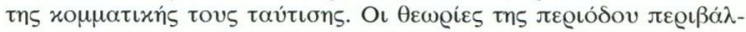

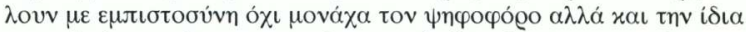

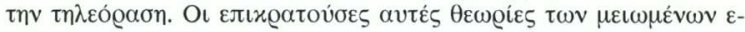

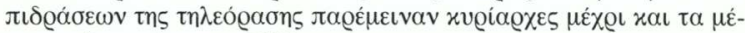

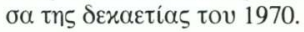

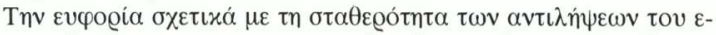

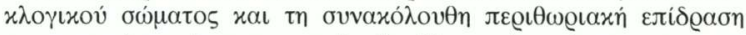

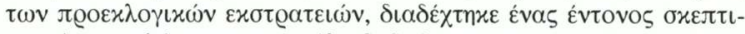

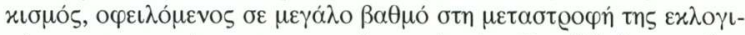

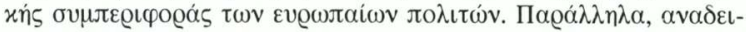

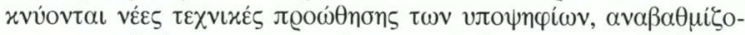

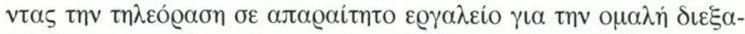

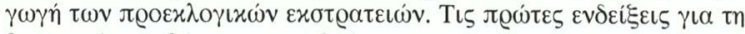

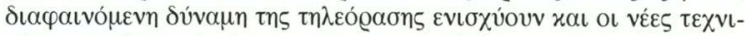

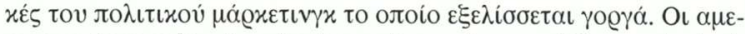

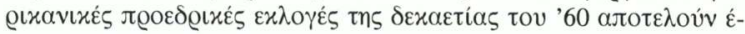

8. G.J. Blumler - D. McQuail, Television in Politics. Its Uses and Influences, Faber

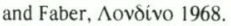

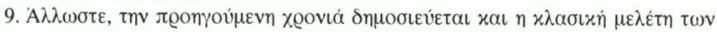
Lipset rau Rokkan, Party Systems and Voter Alignments: Cross-National Perspectives,

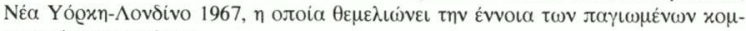

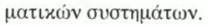




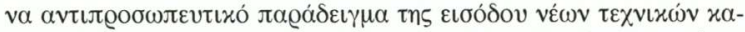

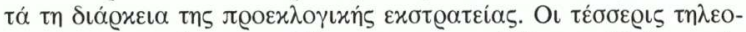

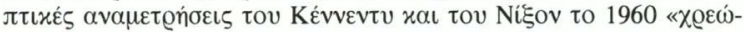

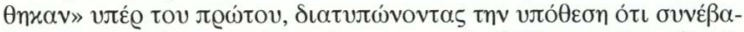

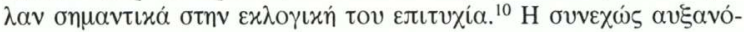

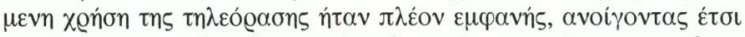

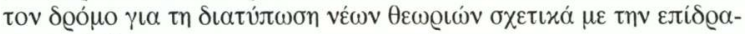

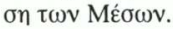

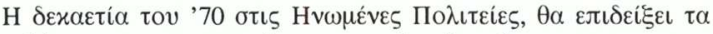

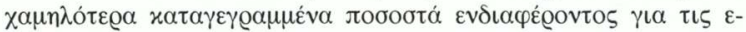

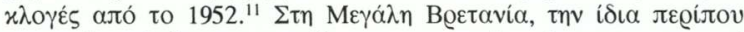

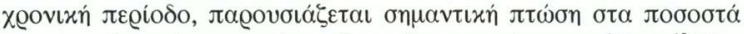

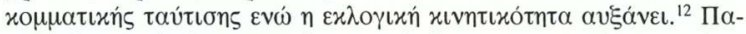

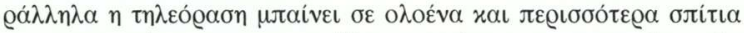

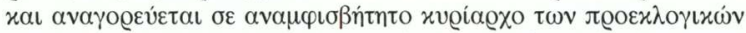

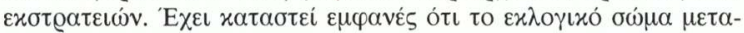

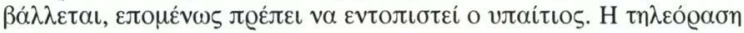

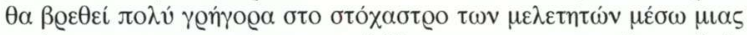

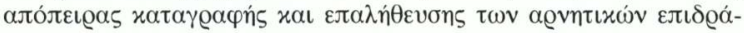

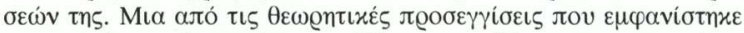

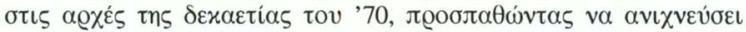

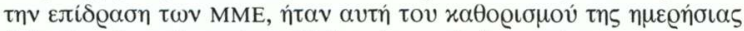

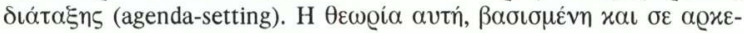

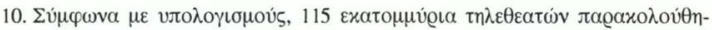

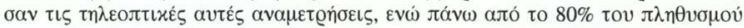

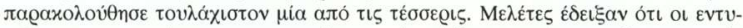

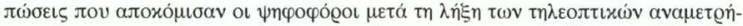

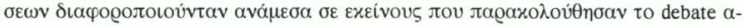

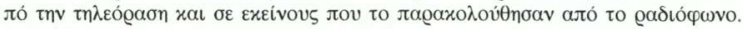

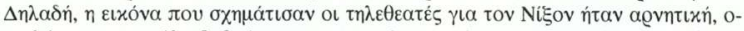

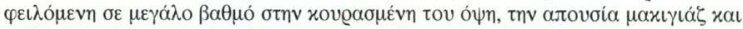

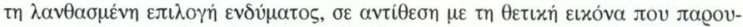

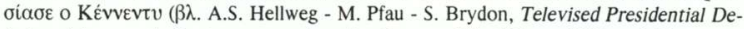
bates. Advocacy in Contemporary America, Praeger, Néa Yó@xך 1992, б. 2-3).

11. B $\lambda$. Ian MacAllister, «Rational or Capricious? Late Deciding Voters in Australia, Britain and the United States», Workshop: «Do Campaigns Matter? The Political Con-

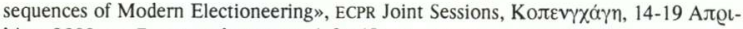

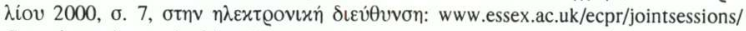
Copenhagen/papers/ws $3 /$ mcallister.PDF

12. B $\lambda$. Anthony Heath $x . \alpha$., Understanding Political Change. The British Voter

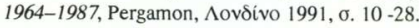




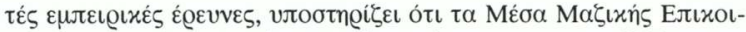

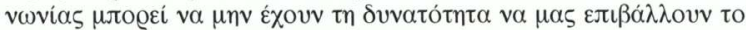

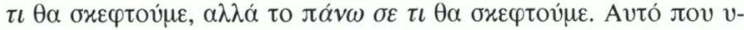

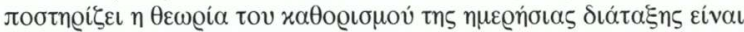

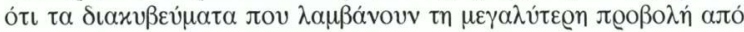

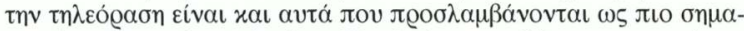

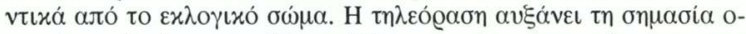

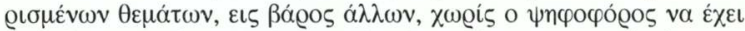

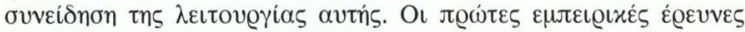

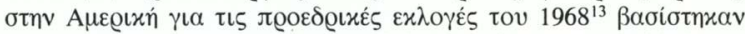

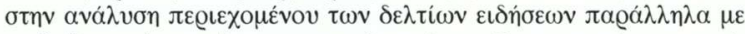

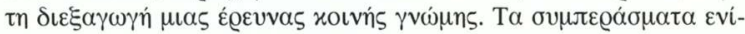

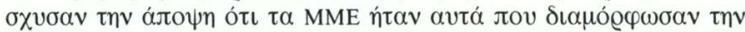

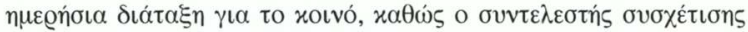

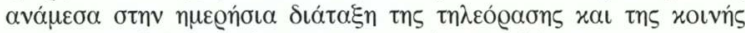

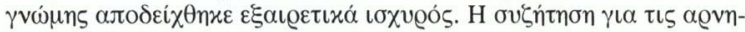

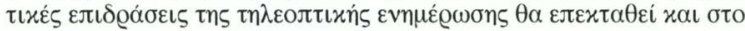

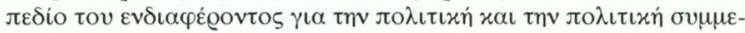

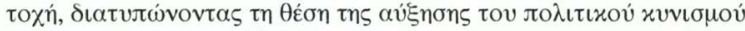

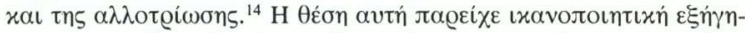

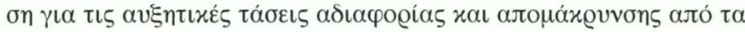

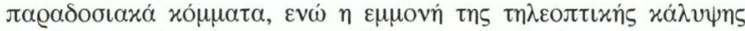

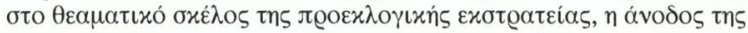

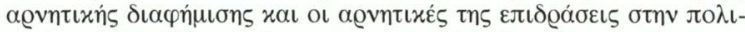

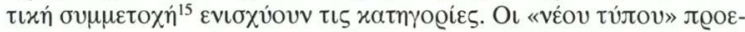

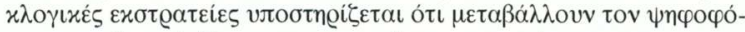

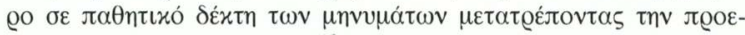

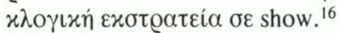

13. M.E. McCombs - D. Shaw, «The Agenda-Setting Function of the Mass Media»,

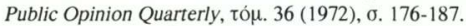

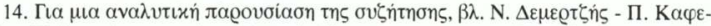

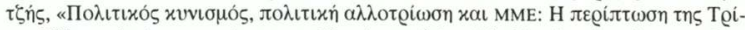

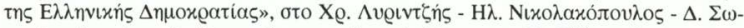

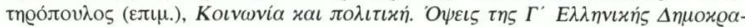

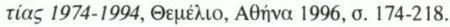

15. S. Ansolabehere - S. Iyengar, Going Negative: How Political Advertisements

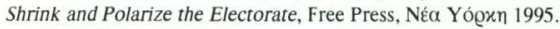

16. Oı Swanson xol Mancini, oto Politics, Media and Modern Democracy. An International Study of Campaign Innovations in Election Campaigning and their Con-

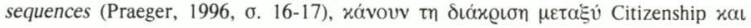


Oı $\theta \varepsilon \omega \varrho i \varepsilon \varsigma ~ \tau \omega \nu ~ \alpha \varrho v \eta \tau \iota x \omega ́ v ~ \varepsilon \pi ı \delta \varrho \alpha ́ \sigma \varepsilon \omega v$ (mediamalaise) $\theta \alpha \pi \alpha \varrho \alpha-$

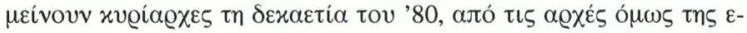

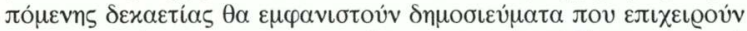

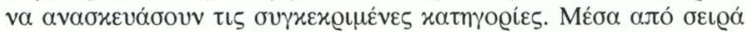

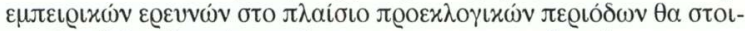

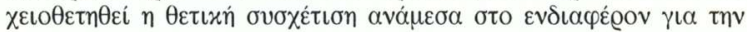

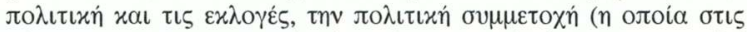

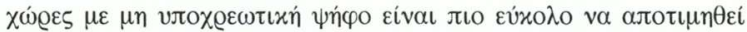

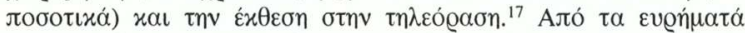

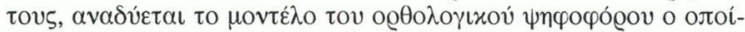

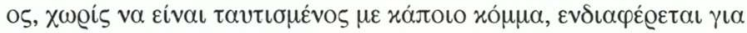

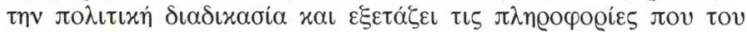

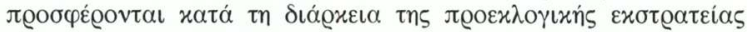

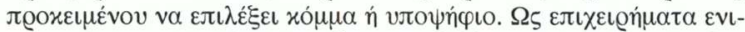

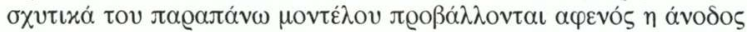

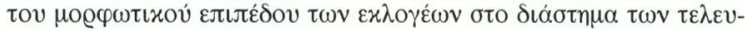

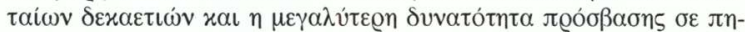

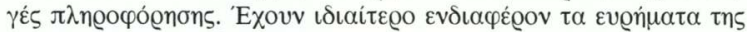

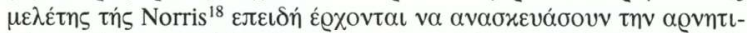

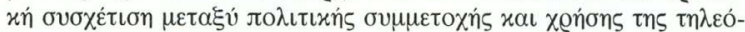

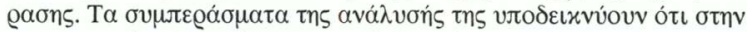

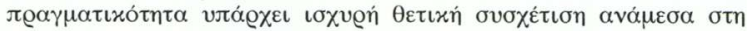

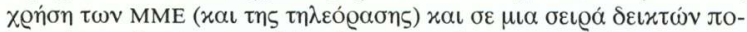

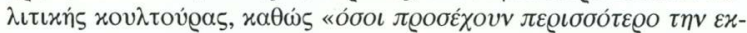

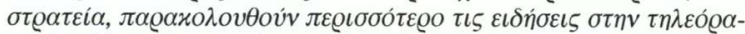

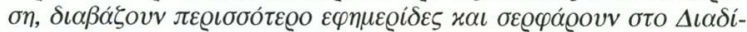

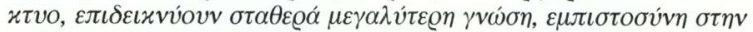

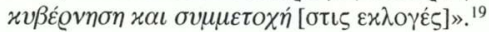

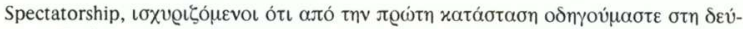

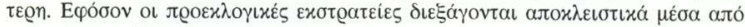

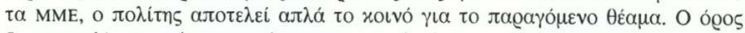

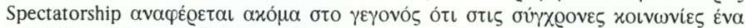

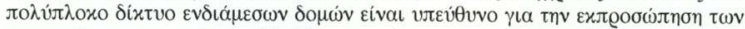

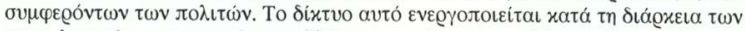

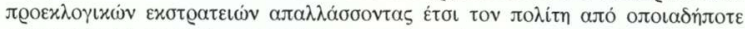

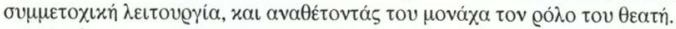

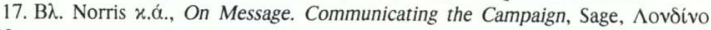
1999.

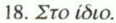

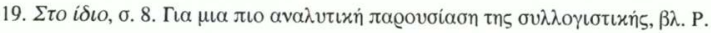




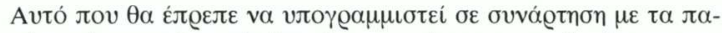

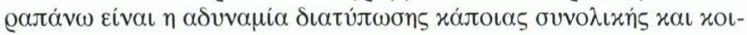

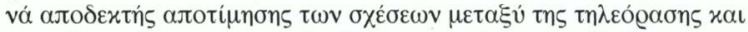

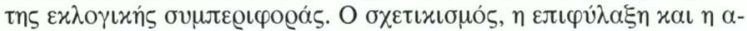

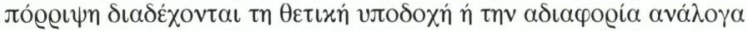

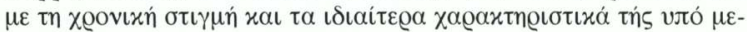

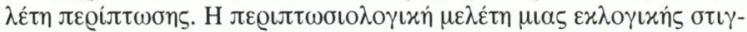

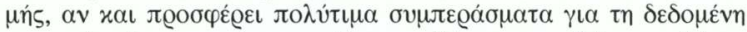

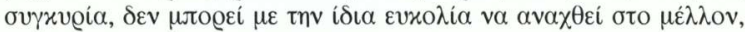

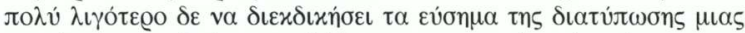

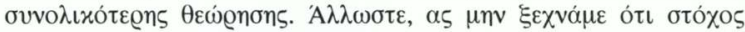

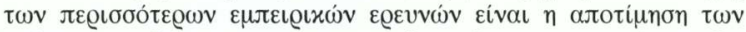

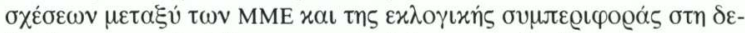

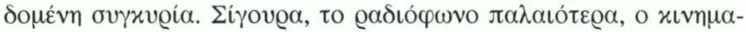

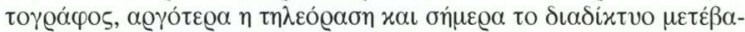

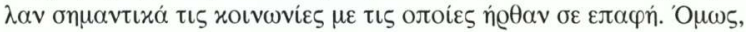
$\eta$ бUvo

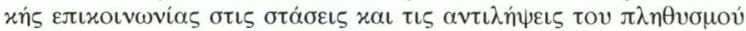

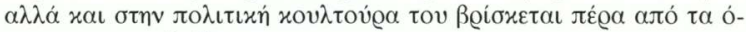

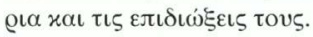

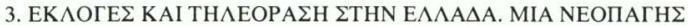 $\Sigma \mathrm{XE} \Sigma \mathrm{H}$}

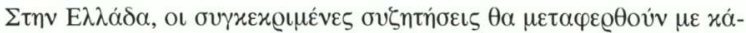

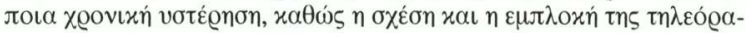

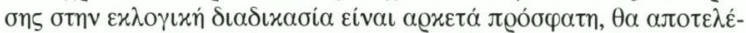

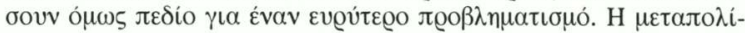

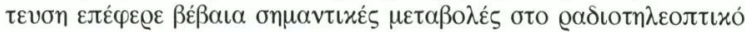

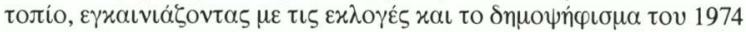

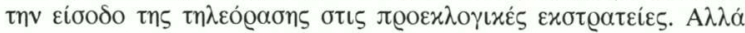

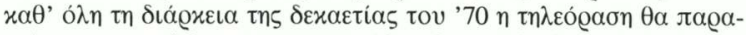

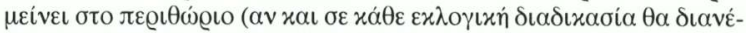

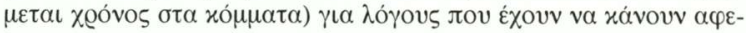

Norris, A Virtuous Circle? Political Communications in Post-Industrial Democracies,

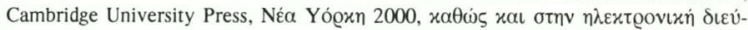
Ouvon http://www.ksg.harvard.edu/people/pnorris/book2.htm. 


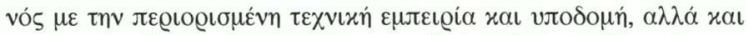

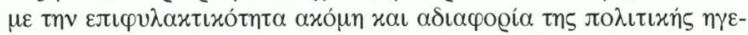

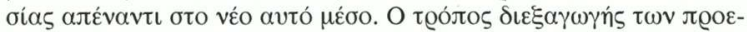

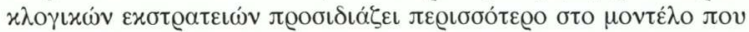

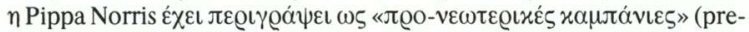

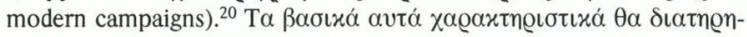

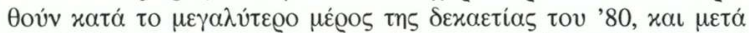

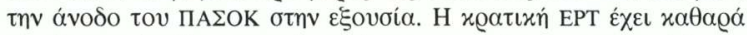

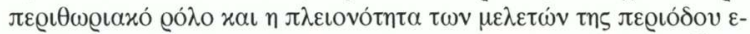

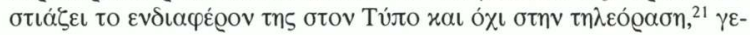

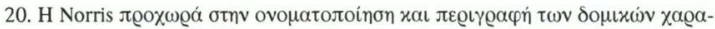

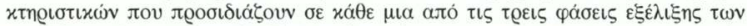

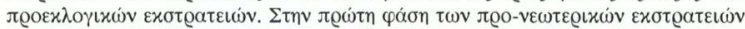

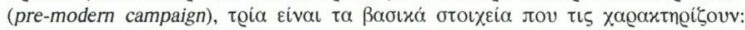

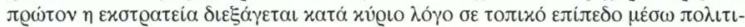

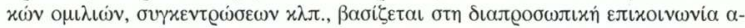
vá

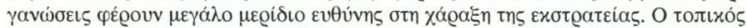

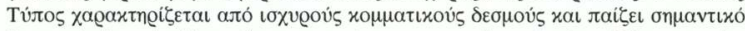

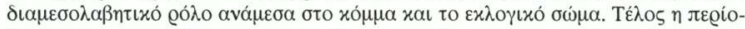

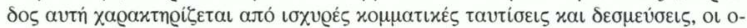

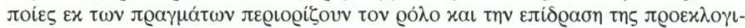

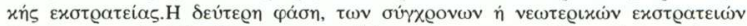

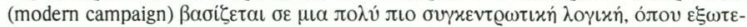

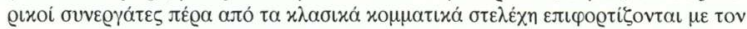

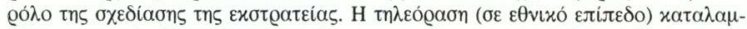

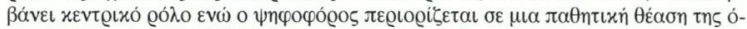

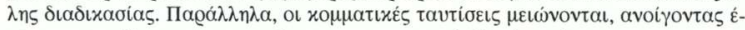

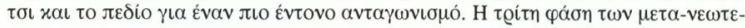

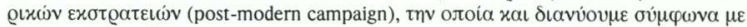

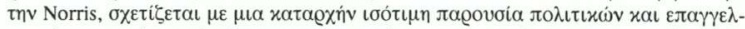

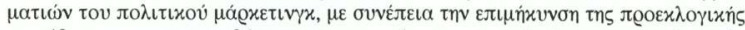

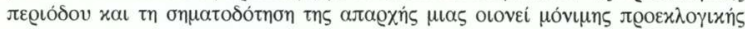

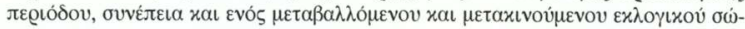

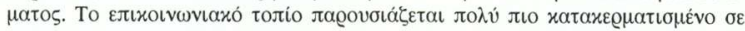

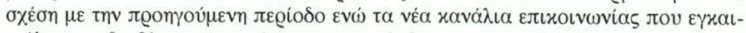

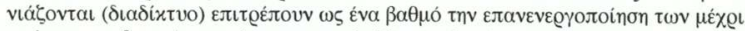

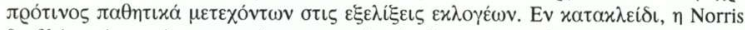

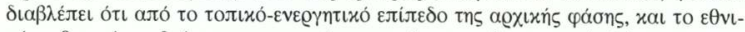

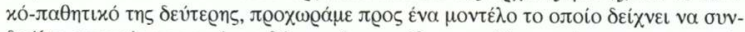

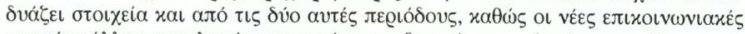

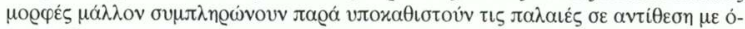

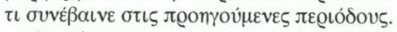

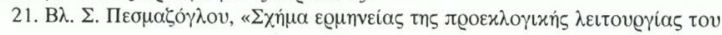




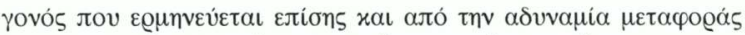

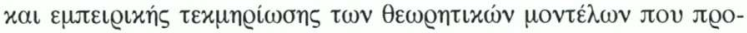

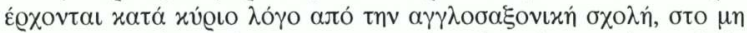

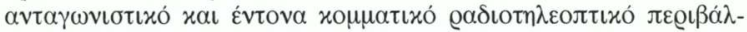

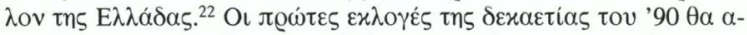

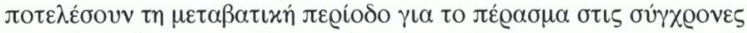

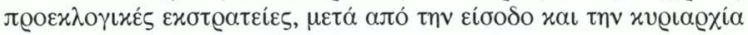

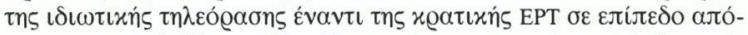

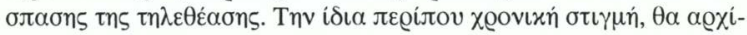

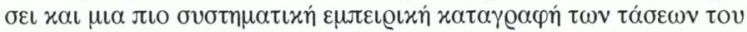

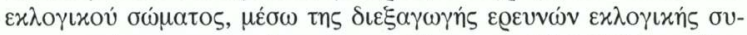

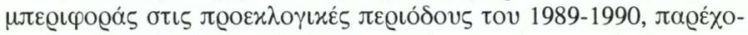

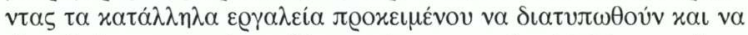

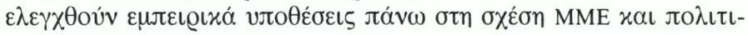

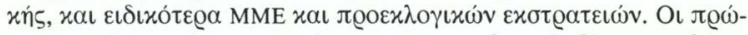

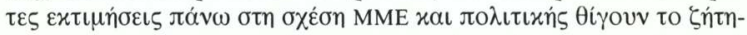

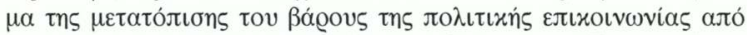

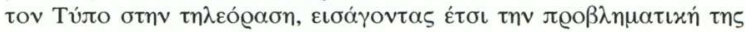

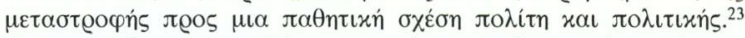

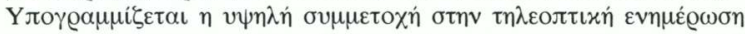

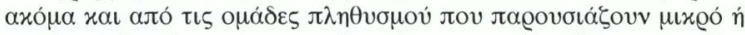

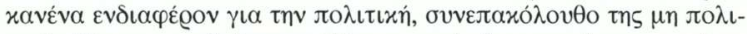

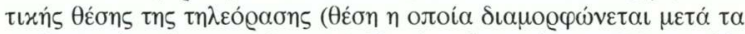

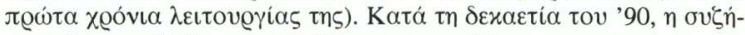

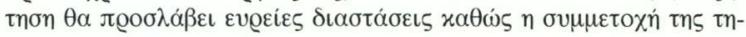

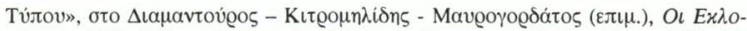

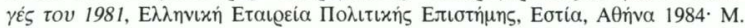

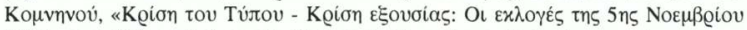

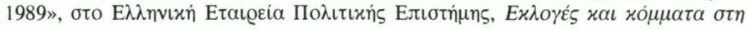

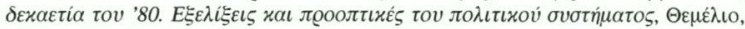
AOńva 1990. Stephanos Pesmazoglou, "The 1980s in the Looking-Glass: PASOK and

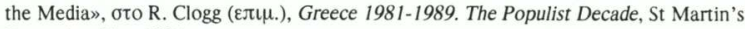

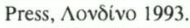

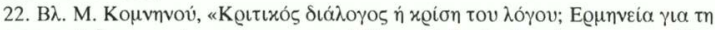

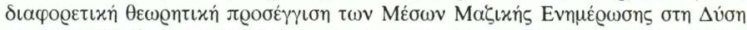

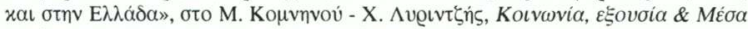

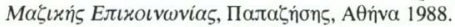

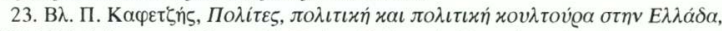

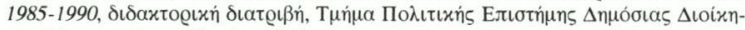

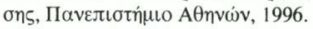




\section{IIINAKA $\mathbf{I}$}

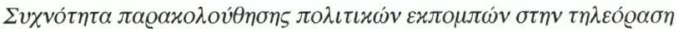
1989-2000*

\begin{tabular}{|c|c|c|c|c|c|c|c|}
\hline & $1989 \alpha$ & $1989 \beta$ & 1990 & 1993 & 1996 & & 2000 \\
\hline 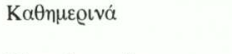 & 65,1 & 69,5 & 74,8 & 75,8 & 74,6 & $\begin{array}{c}\Sigma \chi \varepsilon \delta o ́ v \\
\chi \alpha ́ \theta \varepsilon \mu \varepsilon ́ \varrho \alpha\end{array}$ & 32,3 \\
\hline 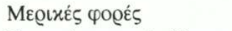 & 20,0 & 20,4 & 16,4 & 13,8 & 10,4 & 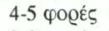 & 15,6 \\
\hline 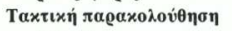 & & & & & & 2-3 Фоฏєً & 22,5 \\
\hline 乏YNOAO & 85,1 & 89,9 & 91,2 & 89,6 & 85,0 & & $\mathbf{7 0 , 4}$ \\
\hline 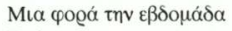 & 3,5 & 2,7 & 1,3 & 3,1 & 5,9 & & \\
\hline$\Sigma \pi \alpha ́ v ı$ & 6,3 & 4,6 & 4,6 & 4,9 & 5,4 & 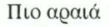 & 18,9 \\
\hline Поте́ & 5,0 & 2,7 & 3,0 & 2,4 & 3,8 & Поте́ & 10,4 \\
\hline \multicolumn{8}{|c|}{ 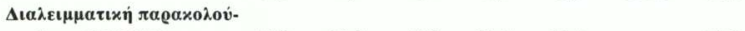 } \\
\hline$\theta \eta \sigma \eta \Sigma Y N O \Lambda O$ & 14,8 & 10,0 & 8,8 & 10,4 & 15,0 & & 29,3 \\
\hline
\end{tabular}

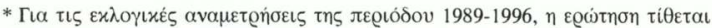

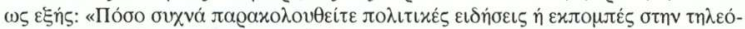

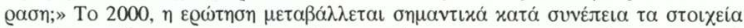

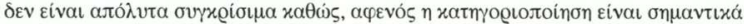

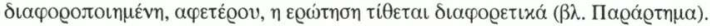

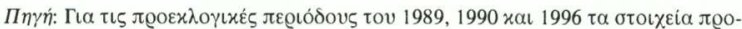

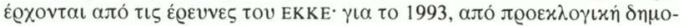

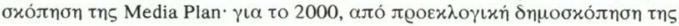
MRB.

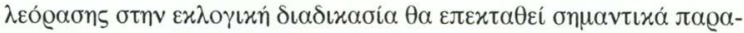

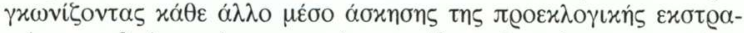

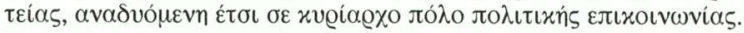

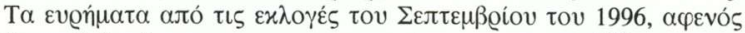

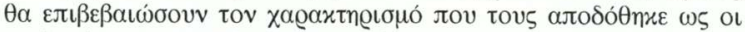

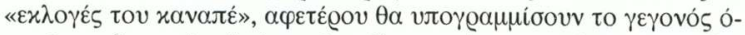

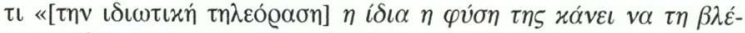

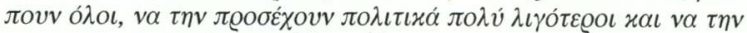

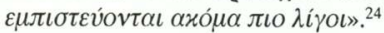

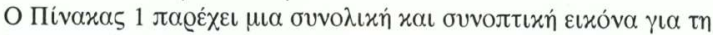

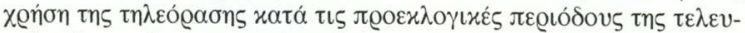

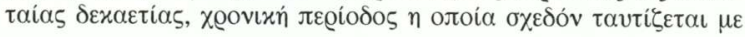

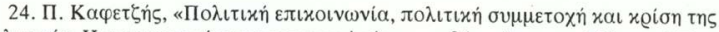

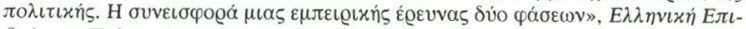

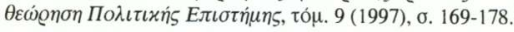




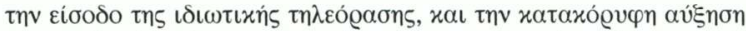

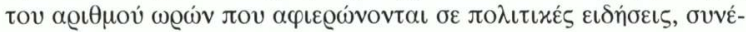

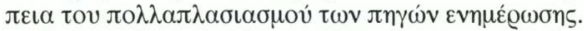

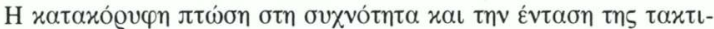

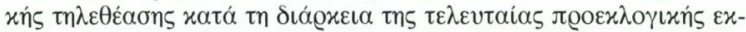

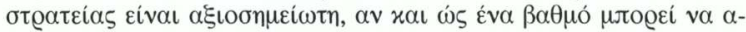

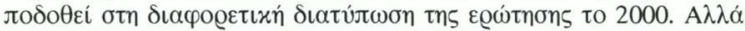

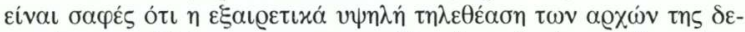

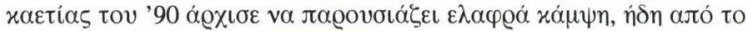

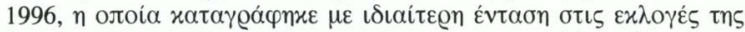

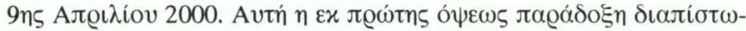

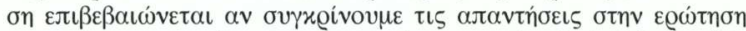

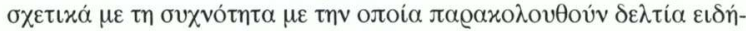

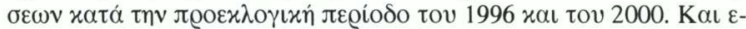

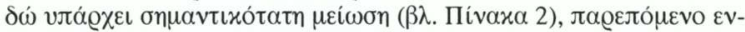

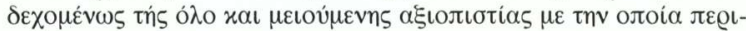

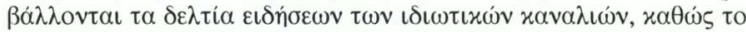

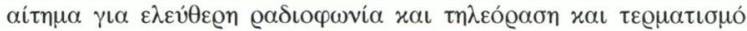

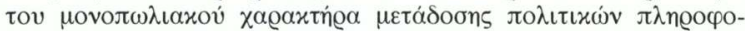

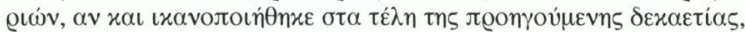

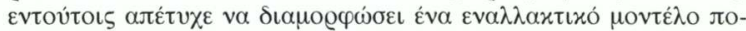

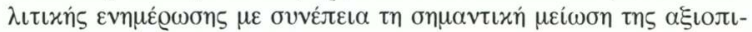

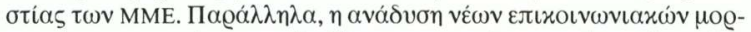

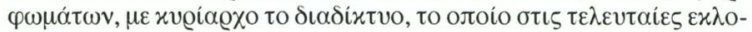

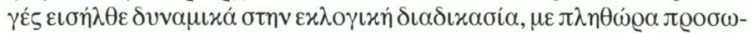

\section{IIINAKA 2}

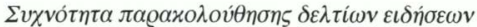

\begin{tabular}{|c|c|c|}
\hline & 1996 & 2000 \\
\hline$\Sigma \chi \varepsilon \delta o ́ v ~ x \alpha ́ \theta \varepsilon \mu \varepsilon \dot{\varepsilon} \varrho \alpha$ & 77,7 & 55,6 \\
\hline 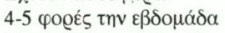 & 13,0 & 16,7 \\
\hline 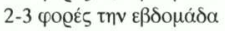 & 8,3 & 16,1 \\
\hline Пıо $\alpha \rho \alpha \iota \alpha$ & 1,0 & 8,2 \\
\hline Поте́* & - & 3,5 \\
\hline
\end{tabular}

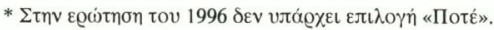

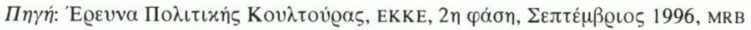

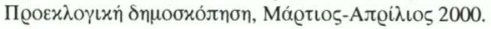




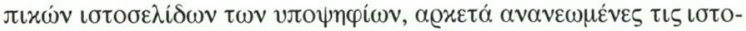

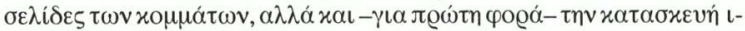

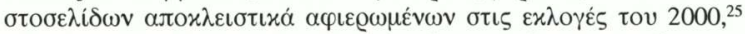

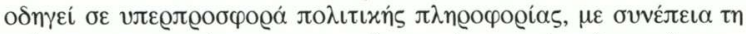

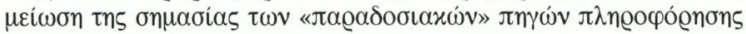

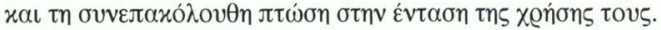

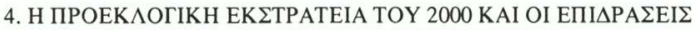 ऽТОN ЧНФОФОРО. МІА ЕМПЕIРІКН КАТАГРАФН}

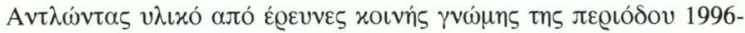

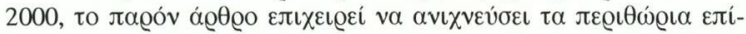

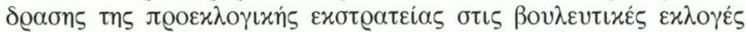

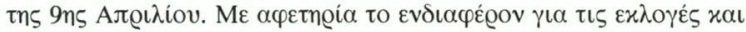

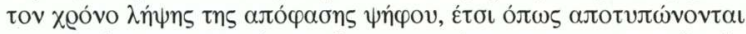

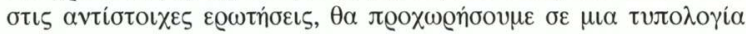

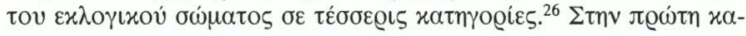

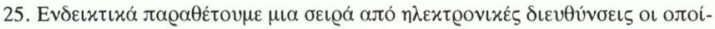

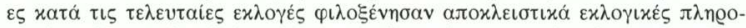

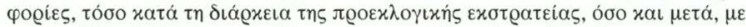

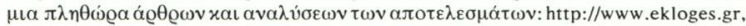
http://www.greekelections.gr, http://www.ekloges2000.gr, http:///www.elections.gr. Av

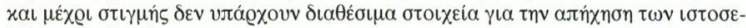

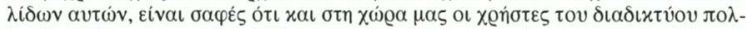

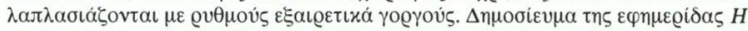

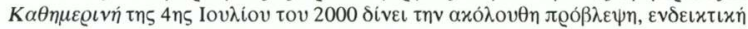

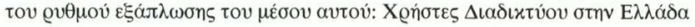

\begin{tabular}{|c|c|}
\hline Etos & 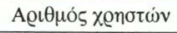 \\
\hline 1998 & 190.000 \\
\hline 1999 & 380.000 \\
\hline 2000 & $800.000^{\circ}$ \\
\hline 2001 & $1.130 .000^{\circ}$ \\
\hline 2002 & $2.000 .000^{\circ}$ \\
\hline 2003 & $2.730 .000^{*}$ \\
\hline 2004 & $3.500 .000^{\circ}$ \\
\hline
\end{tabular}

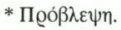

$\Pi \eta \gamma \eta$ : ICAP, Focus-Ban.

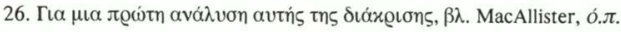




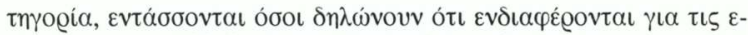

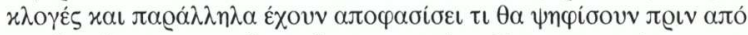

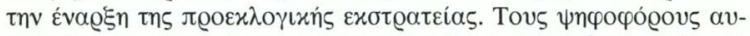

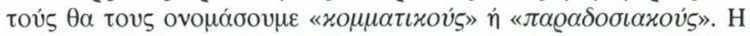

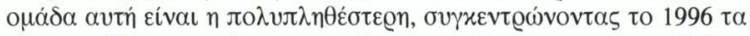

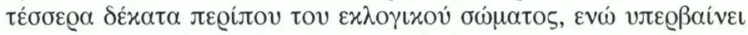

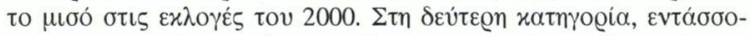

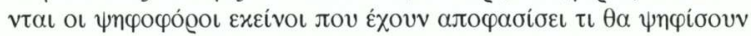

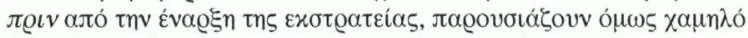

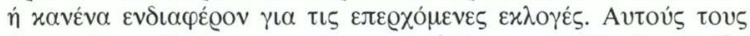

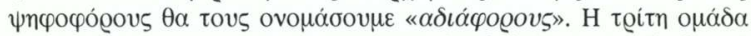

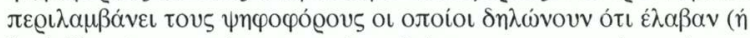

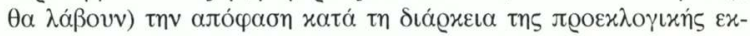

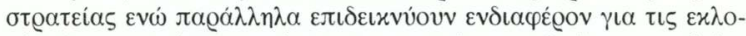

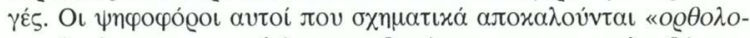

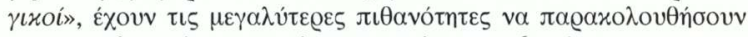

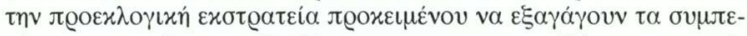

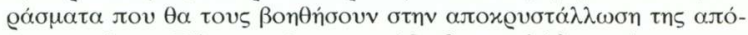

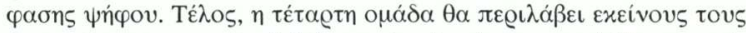

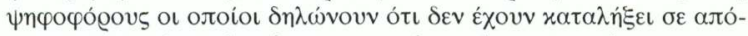

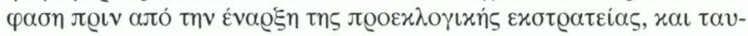

IINAKA 3

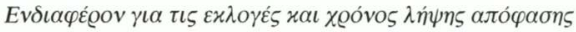

\begin{tabular}{|c|c|c|}
\hline & 2000 & 1996 \\
\hline & & \\
\hline 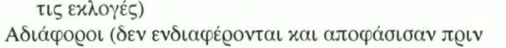 & 52,9 & 40,7 \\
\hline a & 25,1 & 36,2 \\
\hline 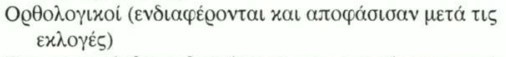 & 9,6 & 9,1 \\
\hline 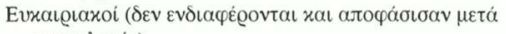 & & \\
\hline$\left.\tau \iota \varsigma \varepsilon x \lambda{ }^{\circ} \gamma \varepsilon \dot{\zeta}\right)$ & 12,4 & 14,0 \\
\hline इYNOAO & 100,0 & 100,0 \\
\hline
\end{tabular}

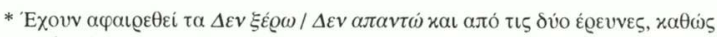

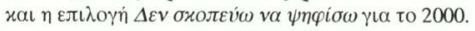

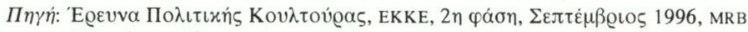

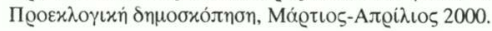




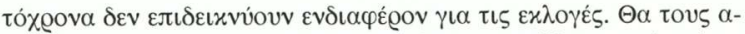

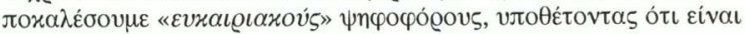

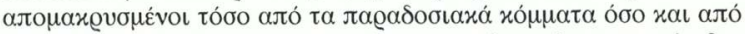

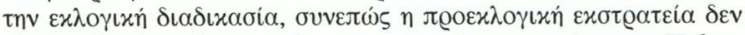

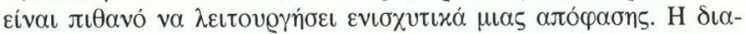

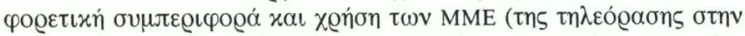

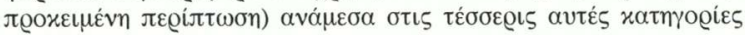

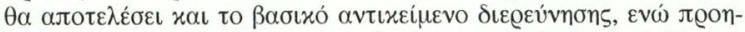

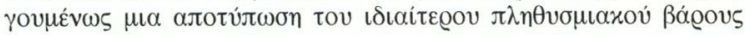

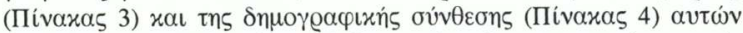

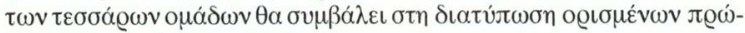
$\tau \omega \nu \pi \alpha \varrho \alpha \tau \eta \emptyset j \sigma \varepsilon \omega v$.

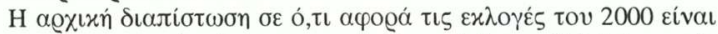

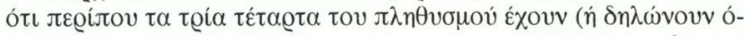

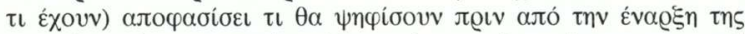

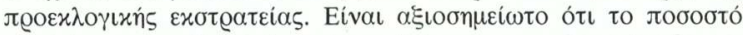

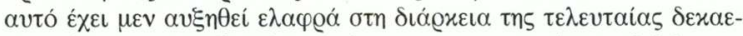

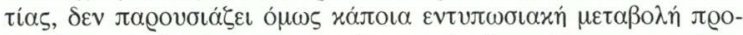

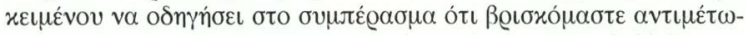

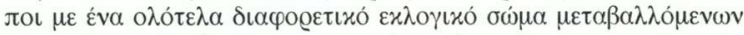

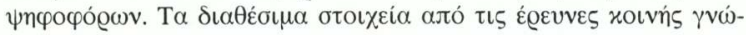

DIAГPAMMA 1

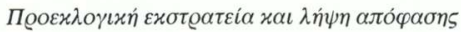

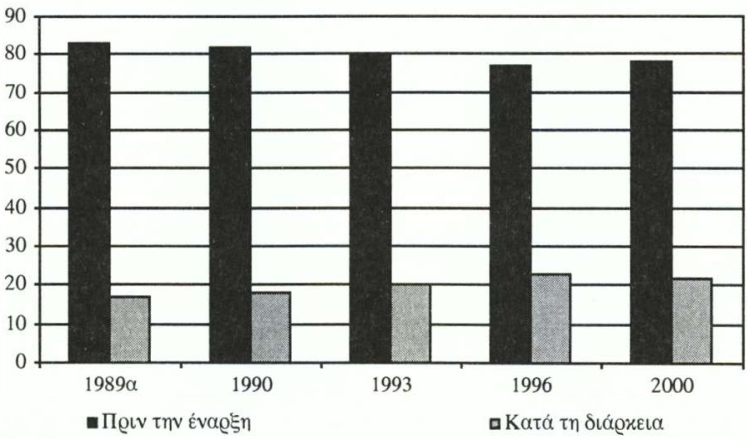




\section{IINAKAs 4}

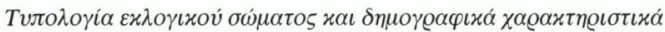

\begin{tabular}{|c|c|c|c|c|c|}
\hline & 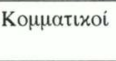 & 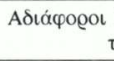 & 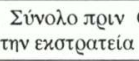 & 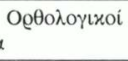 & Evxal@taxoí \\
\hline EYNOAO & 53,0 & 25,1 & 78,1 & 9,6 & 12,4 \\
\hline \multicolumn{6}{|l|}{$\Phi Y \wedge O$} \\
\hline 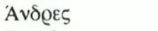 & 59,2 & 20,5 & 79,7 & 9,4 & 10,9 \\
\hline Гvvaixes & 46,6 & 25,1 & 71,7 & 9,7 & 13,9 \\
\hline \multicolumn{6}{|l|}{$H \wedge I K I A$} \\
\hline $18-24$ & 38,3 & 27,2 & 65,7 & 12,8 & 21,8 \\
\hline $25-34$ & 43,3 & 26,7 & 70,0 & 11,8 & 18,2 \\
\hline $35-44$ & 54,0 & 24,8 & 78,8 & 11,5 & 9,7 \\
\hline $45-54$ & 61,0 & 23,7 & 84,7 & 6,5 & 8,1 \\
\hline $55-64$ & 59,0 & 23,3 & 82,2 & 9,0 & 8,7 \\
\hline $65+$ & 62,6 & 24,5 & 87,1 & 5,1 & 7,8 \\
\hline \multicolumn{6}{|l|}{$M O P \Phi \Omega \Sigma H$} \\
\hline $\mathrm{X} \alpha \mu \eta \lambda \dot{n}$ & 56,0 & 28,2 & 74,2 & 7,9 & 7,9 \\
\hline Méon & 50,9 & 26,0 & 76,9 & 8,8 & 14,3 \\
\hline$Y \psi \eta \lambda \dot{n}$ & 52,8 & 18,8 & 71,6 & 13,4 & 15,0 \\
\hline \multicolumn{6}{|c|}{$\psi_{H} \Phi O \Sigma^{*}(\pi \varrho \dot{\theta \varepsilon \sigma \eta})$} \\
\hline ПАЕOK & 60,8 & 26,9 & 87,7 & 7,0 & 5,4 \\
\hline N. $\Delta$. & 61,5 & 26,6 & 88,1 & 5,4 & 6,6 \\
\hline KKE & 56,2 & 29,5 & 85,7 & 5,7 & 8,6 \\
\hline$\Sigma Y N$ & 58,0 & 18,8 & 76,8 & 11,6 & 11,8 \\
\hline$\triangle \mathrm{HKKI}$ & 35,4 & 24,6 & 60,0 & 18,5 & 21,5 \\
\hline$A \wedge \wedge O$ & 23,2 & 16,1 & 39,3 & 28,6 & 32,1 \\
\hline AKYPO/AEYKO & 14,9 & 27,7 & 42,6 & 12,8 & 44,7 \\
\hline
\end{tabular}

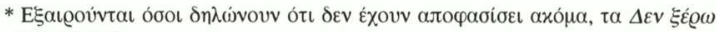
xal $\Delta \varepsilon v \alpha \pi \alpha v \tau \omega ́$.

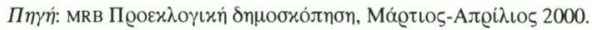

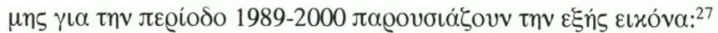

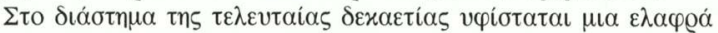

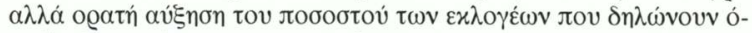

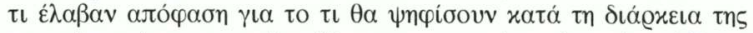

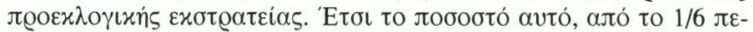

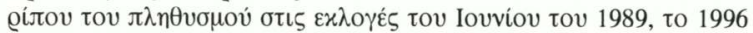

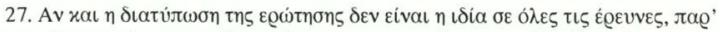

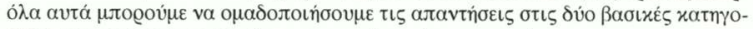

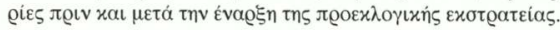




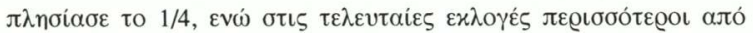

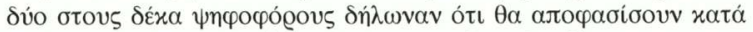

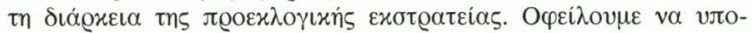

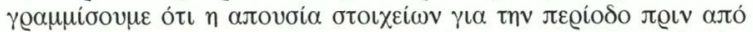

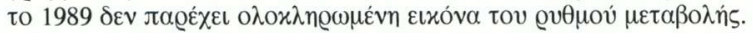

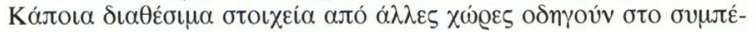

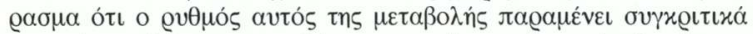

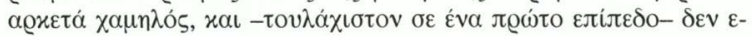

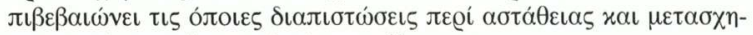

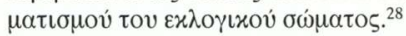

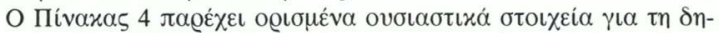

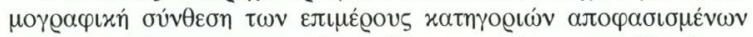

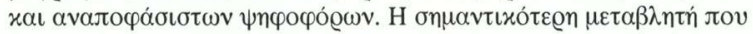

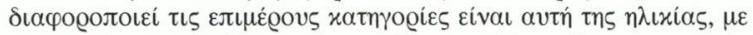

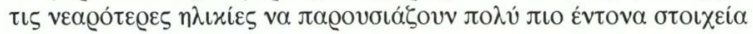

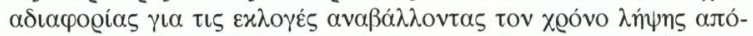

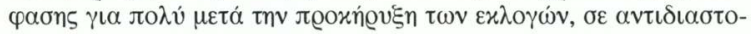

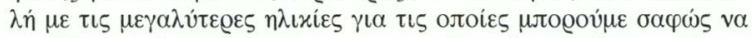

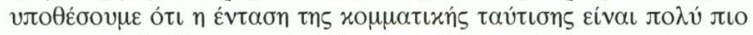

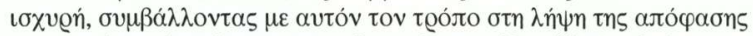

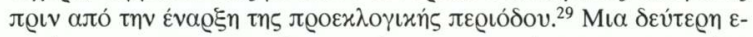

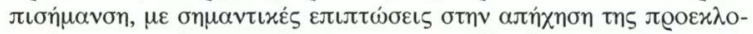

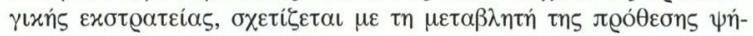

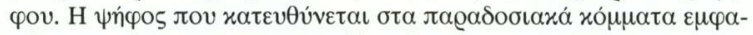

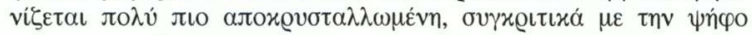

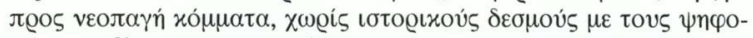

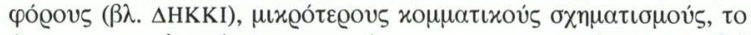

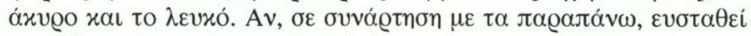

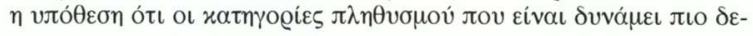

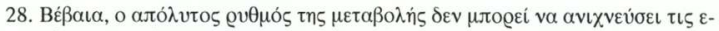

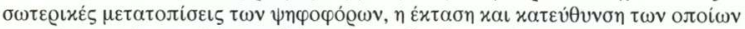

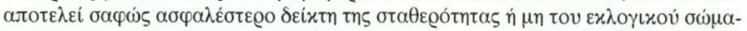

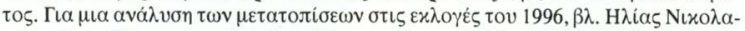

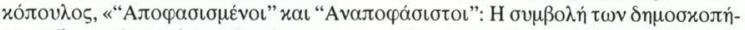

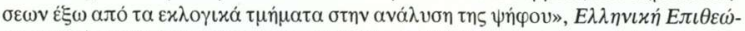

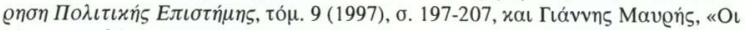

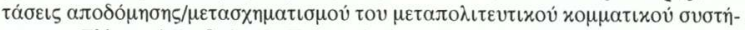

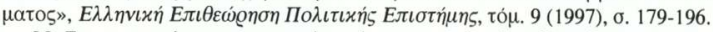

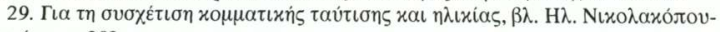
$\lambda 05$, о́.л., б. 202. 


\section{IINAKA 5}

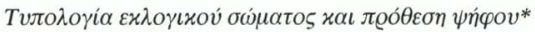

\begin{tabular}{|c|c|c|c|c|c|}
\hline & 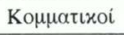 & 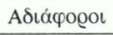 & 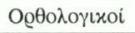 & Evxat@เaxoí & 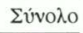 \\
\hline ПАЕOK & 44,9 & 43,0 & 32,9 & 21,9 & 41,0 \\
\hline N. $\Delta$. & 40,7 & 38,1 & 22,8 & 24,0 & 36,8 \\
\hline KKE & 5,8 & 6,6 & 3,8 & 4,9 & 5,8 \\
\hline$\Sigma Y N$ & 4,0 & 2,8 & 5,1 & 4,4 & 3,8 \\
\hline$\triangle \mathrm{HKKI}$ & 2,3 & 3,4 & 7,6 & 7,7 & 3,6 \\
\hline $\mathrm{A} \wedge \wedge \mathrm{O}$ & 1,3 & 1,9 & 10,1 & 9,8 & 3,1 \\
\hline$\triangle E N$ АПОФ. & 0,4 & 1,3 & 13,9 & 15,8 & 3,4 \\
\hline АKYPO/АEYKO & 0,7 & 2,8 & 3,8 & 11,5 & 2,6 \\
\hline$\Sigma Y N O \Lambda O$ & 100,0 & 100,0 & 100,0 & 100,0 & 100,0 \\
\hline
\end{tabular}

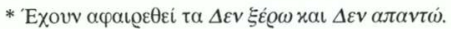

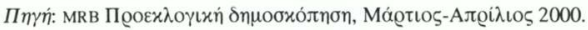

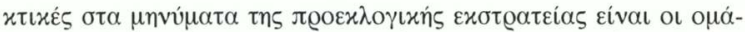

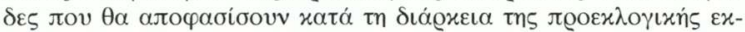

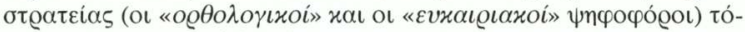

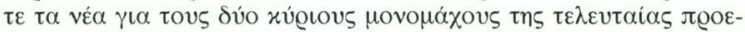

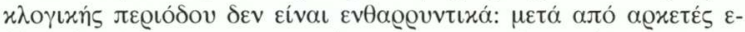

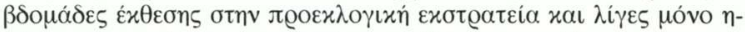

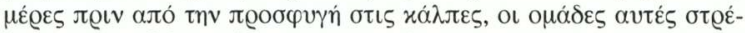

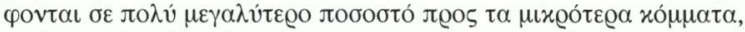

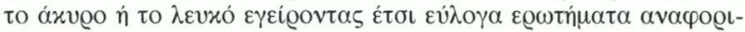

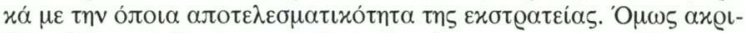

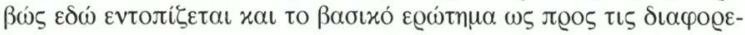

\section{IIINAKAI 6}

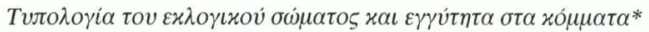

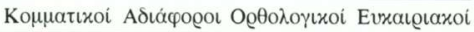

\begin{tabular}{|c|c|c|c|c|}
\hline 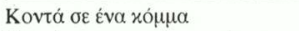 & 76,7 & 72,3 & 32,7 & 29,6 \\
\hline 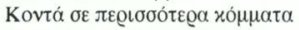 & 18,7 & 15,4 & 36,3 & 21,4 \\
\hline 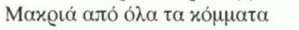 & 4,6 & 12,3 & 31,0 & 49,0 \\
\hline$\Sigma Y N O \wedge O$ & 100,0 & 100,0 & 100,0 & 100,0 \\
\hline
\end{tabular}

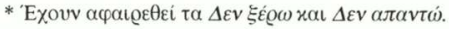

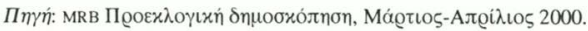




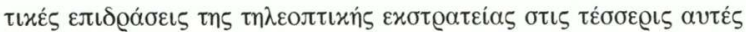

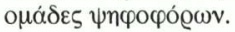

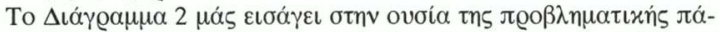
v

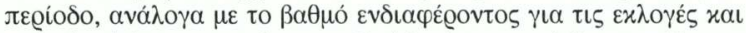

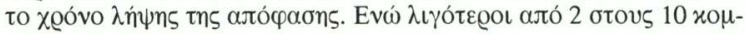

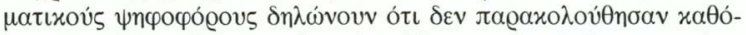

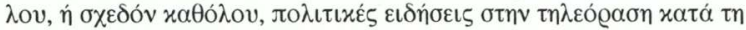

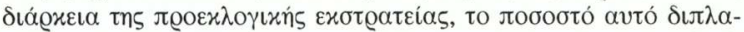

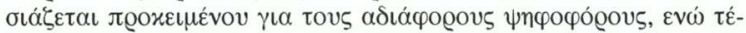

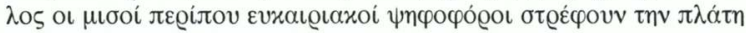

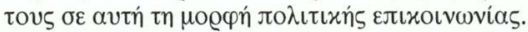

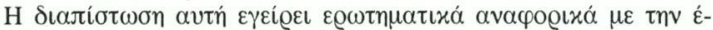

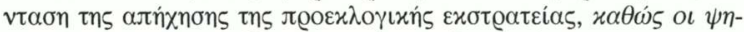

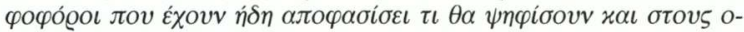

\section{МIAГPAMMA 2}

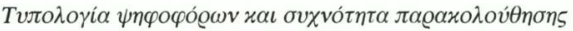
$\pi 0 \lambda \iota \tau \iota \varkappa \omega ́ v \varepsilon \iota \delta \dot{\eta} \sigma \varepsilon \omega v$

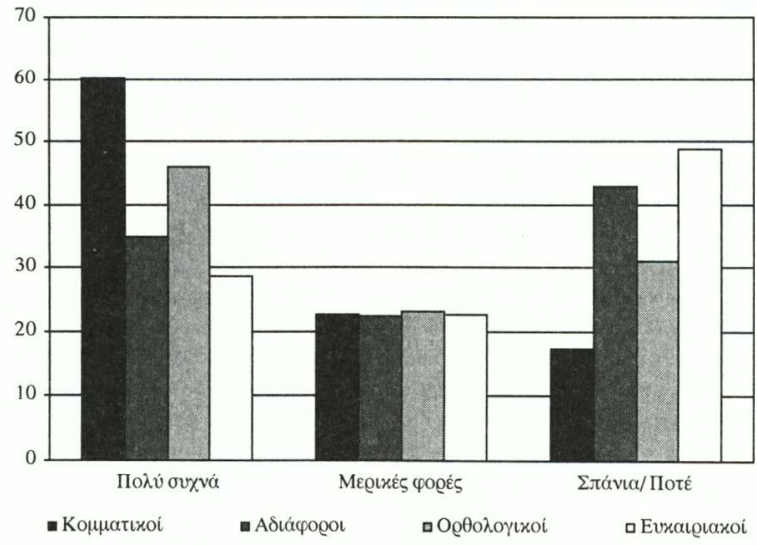

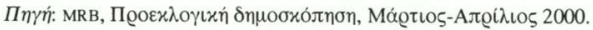




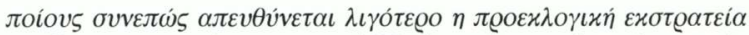

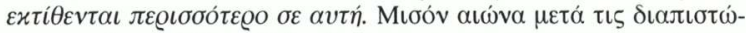

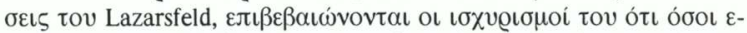

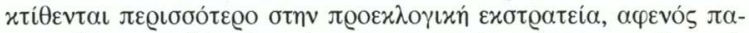

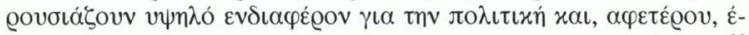

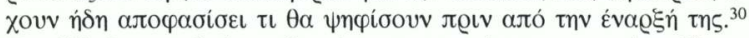

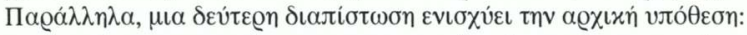

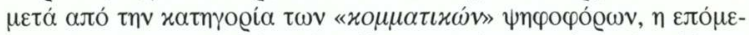

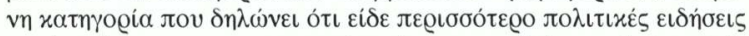

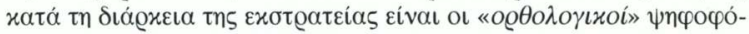

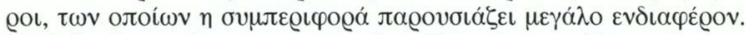

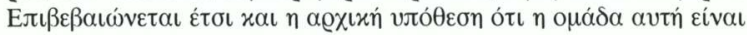

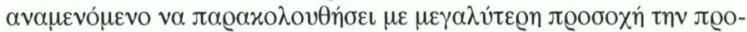

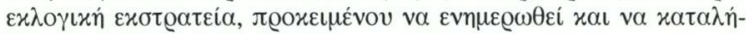

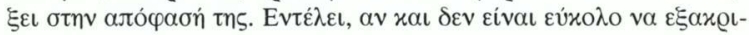

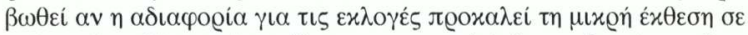

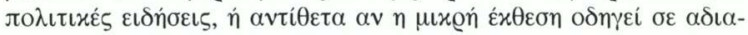

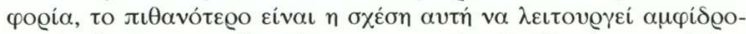

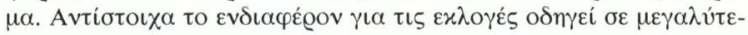

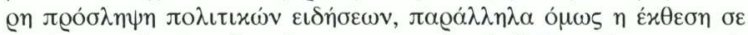

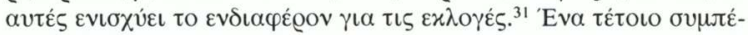

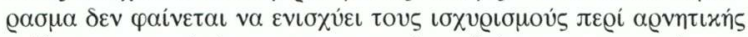

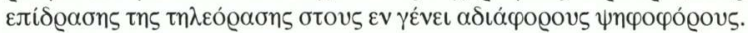

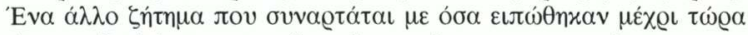

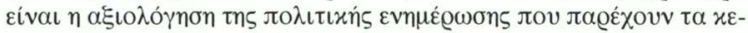

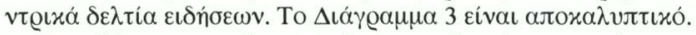

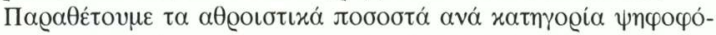

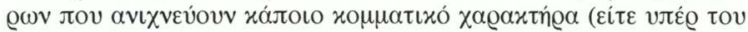

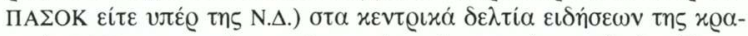

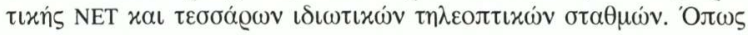

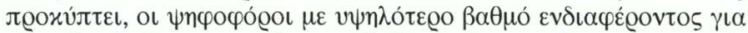

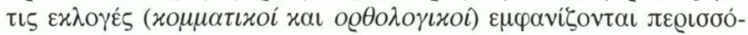

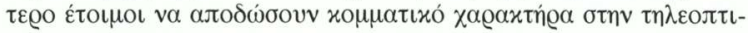

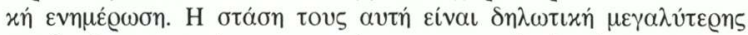

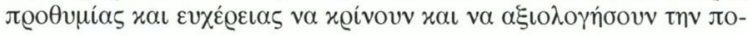

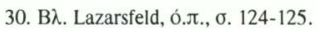

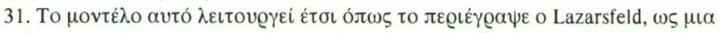

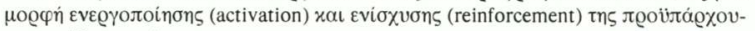

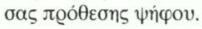


МIAГPAMMA 3

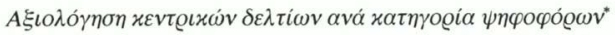

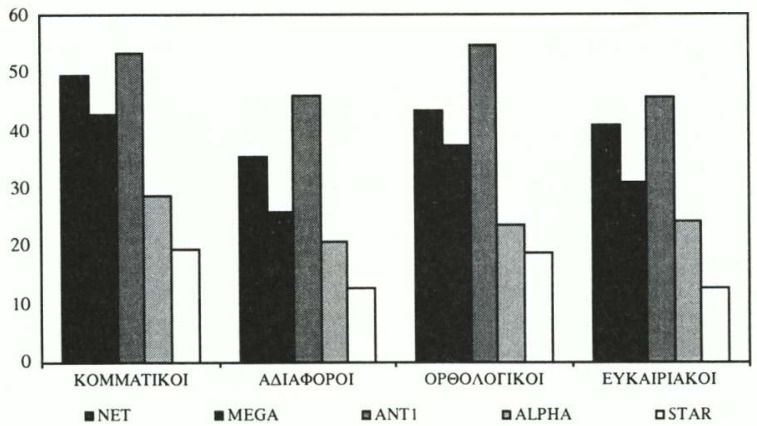

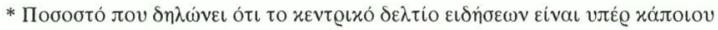
xó $\mu \mu \alpha \tau o s$.

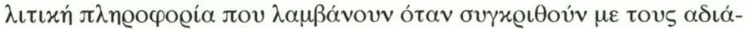

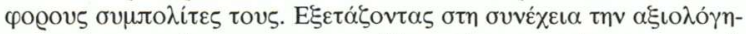

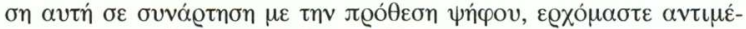

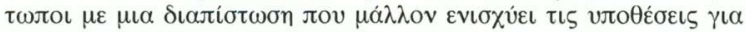

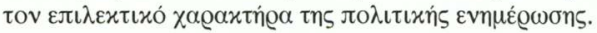

\section{IIINAKA 7}

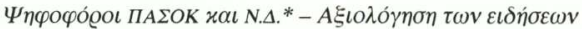
$\pi \circ v \pi \alpha \varrho \alpha$ ж)

\begin{tabular}{|c|c|c|c|c|c|}
\hline \multicolumn{3}{|c|}{ 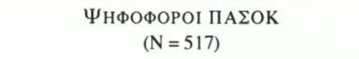 } & \multicolumn{3}{|c|}{$\begin{array}{l}\Psi_{\text {НФОФОРОI N. }} . \\
\qquad(\mathrm{N}=415)\end{array}$} \\
\hline 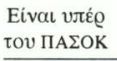 & 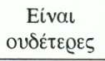 & 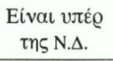 & 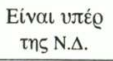 & 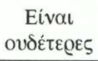 & 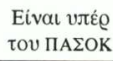 \\
\hline 36,36 & 47,58 & 16,05 & 39,03 & 40,72 & 20,24 \\
\hline
\end{tabular}

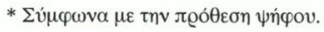

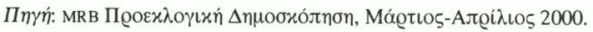




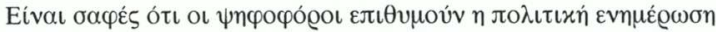

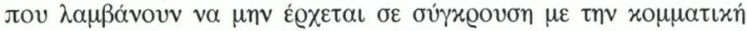

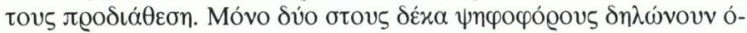

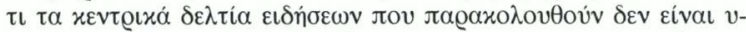

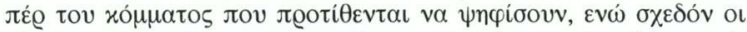

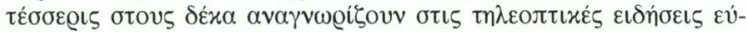

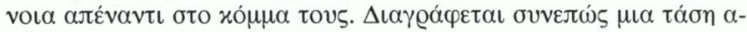

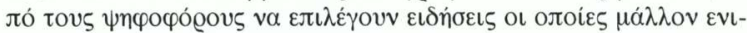

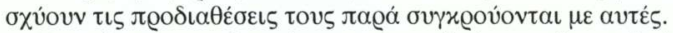

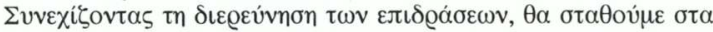

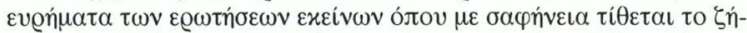

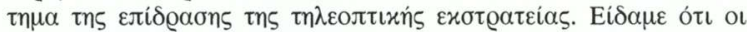

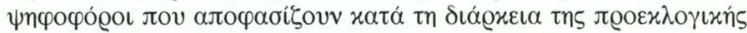

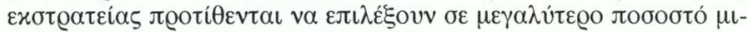

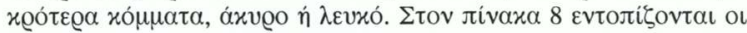

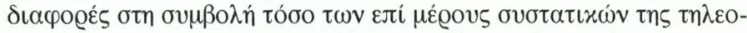

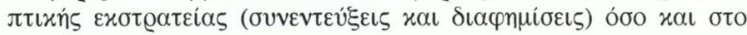
бúvoגo tns.

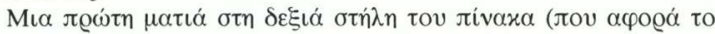

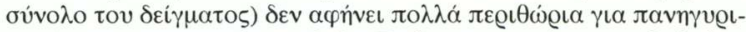

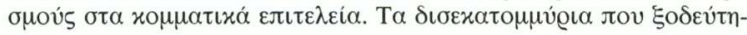

\section{IINAKAE 8}

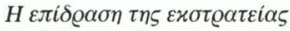

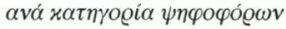

\begin{tabular}{|c|c|c|c|c|c|}
\hline & 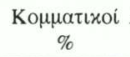 & $\begin{array}{c}\text { Adı́x } \% \text { ọot } \\
\%\end{array}$ & $\begin{array}{c}\text { O@Өодоүเхоі } \\
\%\end{array}$ & $\begin{array}{c}\text { Evralguxoi } \\
\%\end{array}$ & 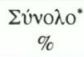 \\
\hline \multicolumn{6}{|c|}{$\Sigma v \mu \beta 0 \lambda \eta \dot{~ \Sigma v v \varepsilon v \tau \varepsilon v ์ \xi \xi \varepsilon \omega v ~}$} \\
\hline 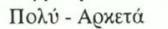 & 17,3 & 7,1 & 21,1 & 7,9 & 14,0 \\
\hline$\Lambda i \gamma o-K a \theta \dot{\lambda} \lambda o v$ & 82,7 & 92,9 & 78,9 & 92,1 & 86,0 \\
\hline \multicolumn{6}{|c|}{$\Sigma v \mu \beta 0 \lambda \eta \dot{\eta} \Delta \iota \alpha \varphi \eta \mu i \sigma \varepsilon \omega v$} \\
\hline Подט́ - Аดxєtá & 6,8 & 4,2 & 6,5 & 2,1 & 5,5 \\
\hline$\Lambda i \gamma o-\mathrm{K} \alpha \theta \dot{\lambda} \lambda \circ v$ & 93,2 & 95,8 & 93,5 & 97,9 & 94,5 \\
\hline \multicolumn{6}{|c|}{ 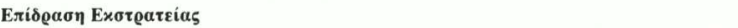 } \\
\hline 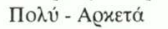 & 12,0 & 4,6 & 16,1 & 5,0 & 9,7 \\
\hline 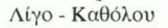 & 88,0 & 95,4 & 83,9 & 95,0 & 90,3 \\
\hline
\end{tabular}

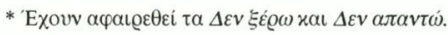

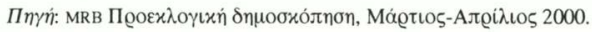




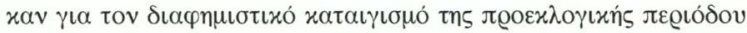

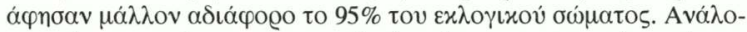

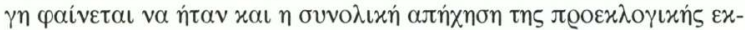

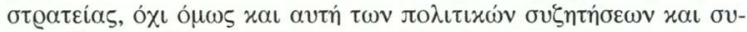

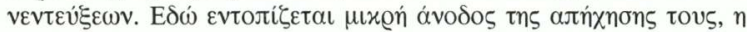

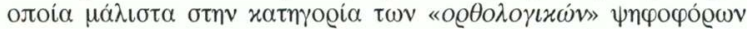

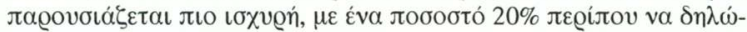

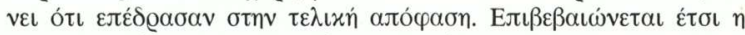

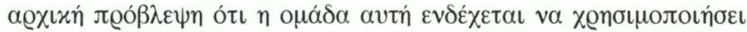

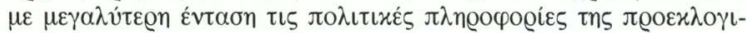

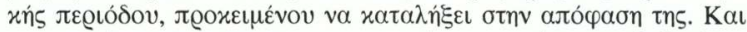

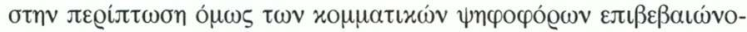

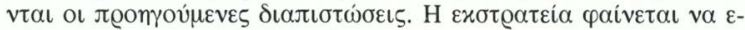

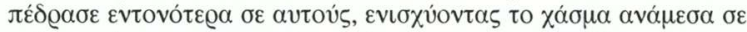

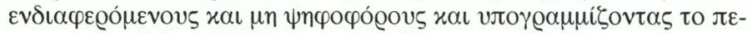

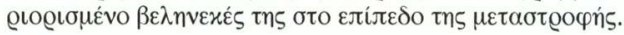

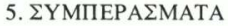

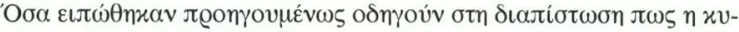

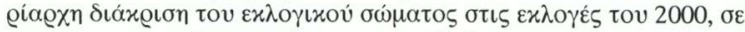

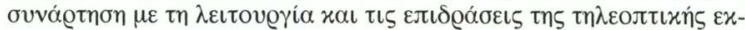

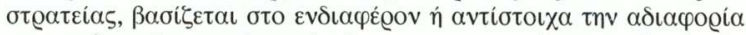

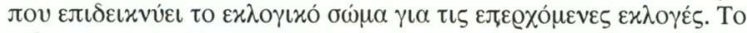

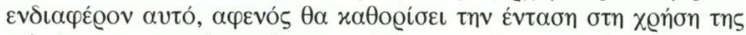

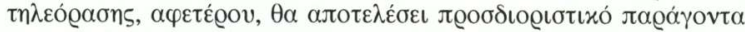

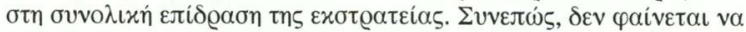

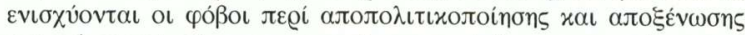

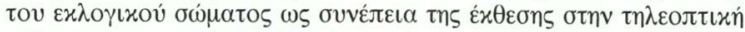

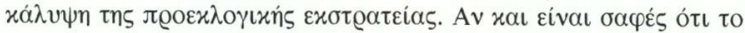

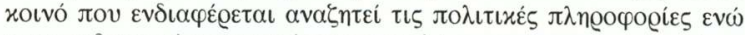

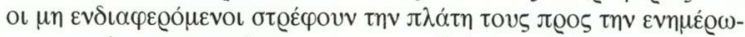

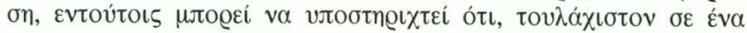

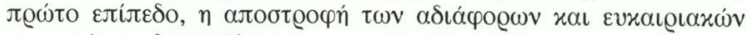

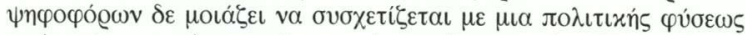

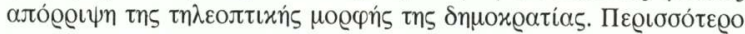

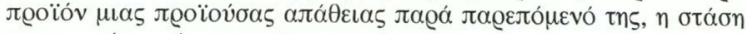

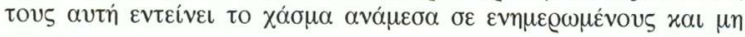




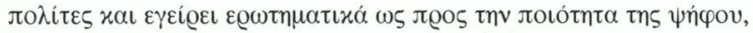

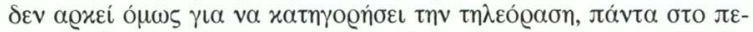

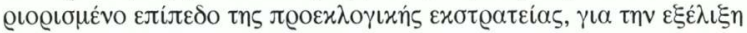

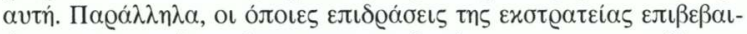

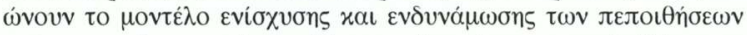

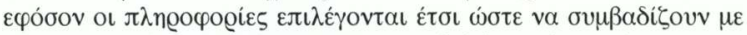

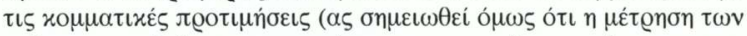

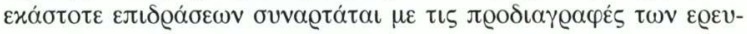

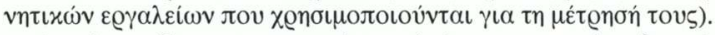

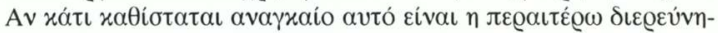

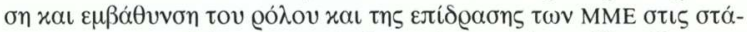

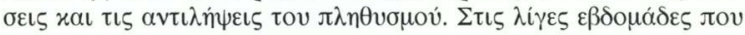

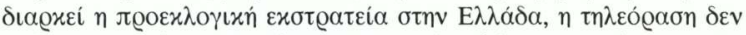

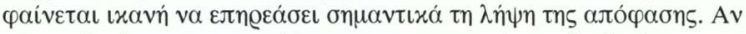

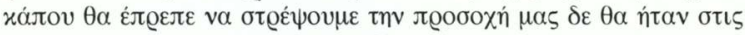

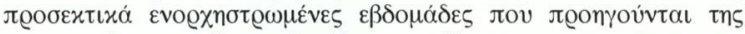

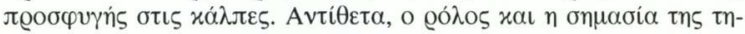

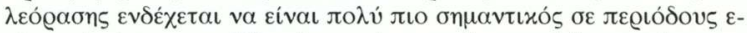

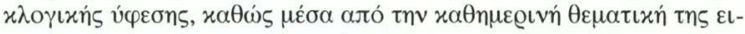

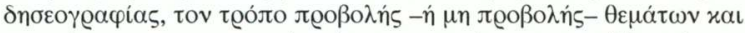

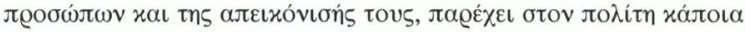

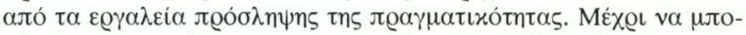

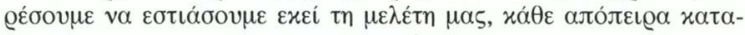

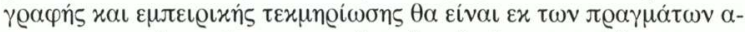

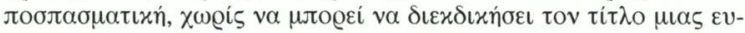

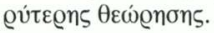




\section{ПАРАРТНМА}

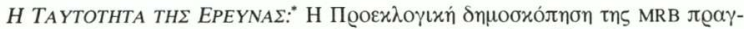

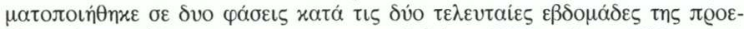

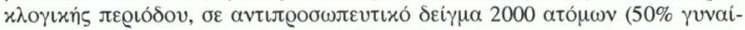

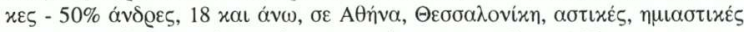

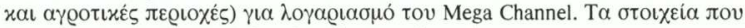

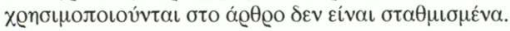

\section{EPSTHEH 3}

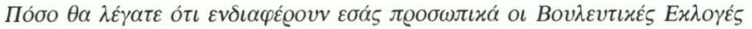

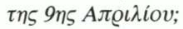
ПО $\Lambda \mathrm{Y}$
APKETA
$\Lambda \mathrm{IIO}$

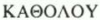

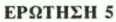

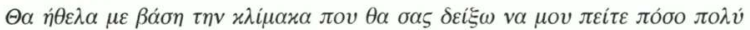

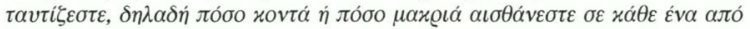
$\tau \alpha$ жó $\mu \alpha \tau \alpha \pi \circ v \theta \alpha \sigma \alpha \varsigma \delta \iota \alpha \beta \alpha \alpha \omega$.

\section{EPSTHEH 13}

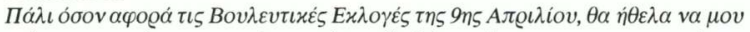

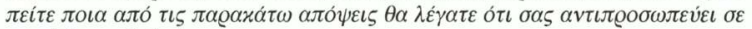

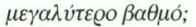

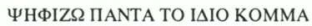

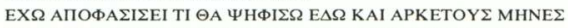

EX $\Omega$ AПОФA $\Sigma I \Sigma E I T I ~ \Theta A ~ \Psi H \Phi I \Sigma \Omega$ E $\Delta \Omega$ KAI MEPIKE $\Sigma$ EB $\triangle O M A \triangle E \Sigma$

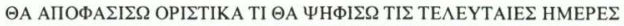

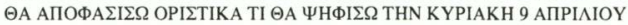

\section{EPSTHEH 16 $\alpha$}

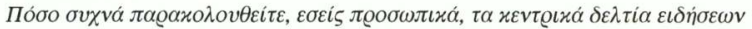

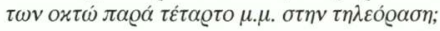

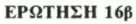

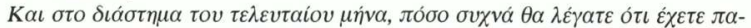

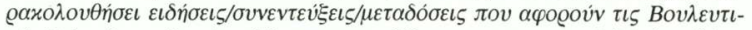

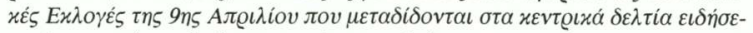

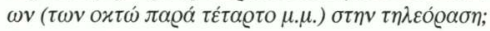

$X E \triangle O N$ KAOE MEPA

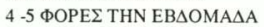

$2-3$ ФOPE $\Sigma$ THN EB $\triangle O M A \triangle A$

ПIO APAIA

ПIОТЕ

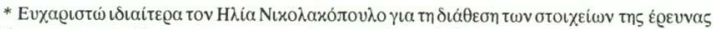

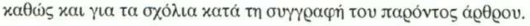




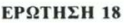

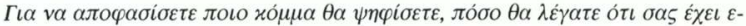

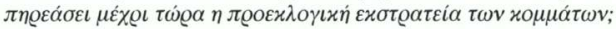

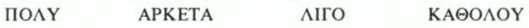

\section{EPSTHЕH $19 \alpha$}

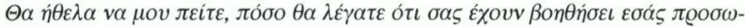

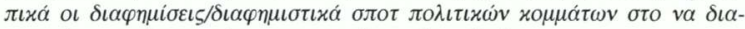

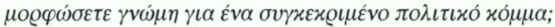

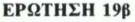

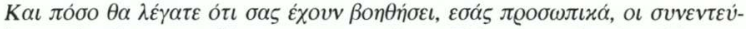

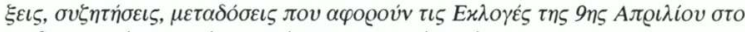

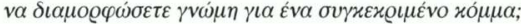

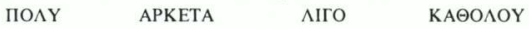

\section{EPSTHЕH 24}

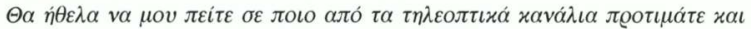

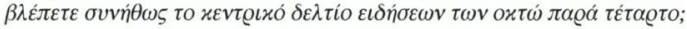

\section{EPQTHгH 25}

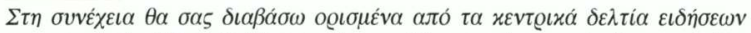

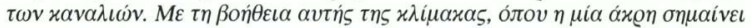

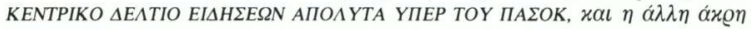

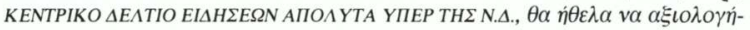

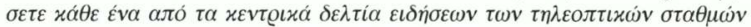

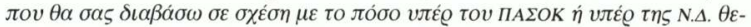

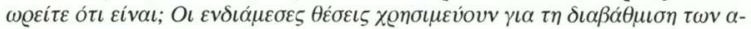
$\pi \delta ́ \psi \varepsilon \omega v \sigma \alpha \varsigma$.

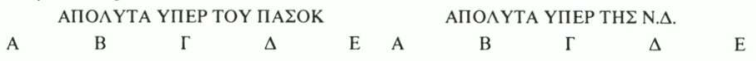

\section{BIBАIОГРАФІA}

ANSOlabehere, S. - IYengar, S. (1995), Going Negative: How Political Advertisements Shrink and Polarize the Electorate, Free Press, Néa Yógxn.

BARTOlini, S. - MAIR, P. (1990), Identity, Competition and Electoral Availability, Cambridge University Press, Cambridge.

BERELSON, B. - LAZARSFEld, P. - MCPHEE, W. (1954), Voting: A Study of Opinion Formation in a Presidential Campaign, Chicago University Press,

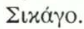


BLUMLeR, G.J. - MCQUAIL, D. (1968), Television in Politics. Its Uses and

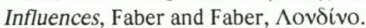

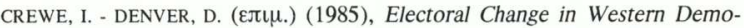

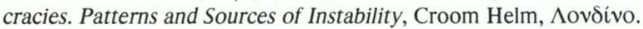

FRANKLIN - MACKIE - VALEN $x$.ó. (1992), Electoral Change: Responses to Evolving Social and Attitudinal Structures in Western Europe, Cambridge University Press, Cambridge.

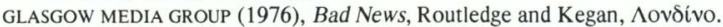

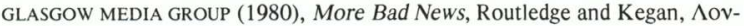
Sívo.

GLASGOW MEDIA GROUP (1982), Really Bad News, Routledge and Kegan,

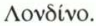

heath, A. - JOWEll, R. - Curtice, J. (1991), Understanding Political Change.

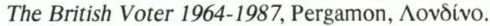

HellweG, A.S. - PFAU, M. - BRYdON, S. (1992), Televised Presidential Debates.

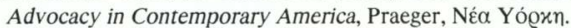

KLAPPER, J. (1960), The Effects of Mass Communication, Free Press, Né $\alpha$ Yógxn.

LANE, J. E. - ERSSON, S. (1994), Politics and Society in Western Europe, Sage,

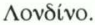

LAZARSFEld, F.P. - BERELSON, B. - GAUdeT, H. (1948), The People's Choice. How the Voter Makes up his Mind in a Presidential Campaign, Columbia

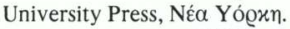

LIPSET, S. - ROKKAN, S. (1967), Party Systems and Voter Alignments: Cross-

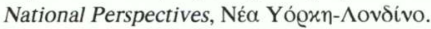

MACAllister, I. (2000), «Rational or Capricious? Late Deciding Voters in Australia, Britain and the United States», Workshop: «Do Campaigns Matter? The Political Consequences of Modern Electioneering», ECPR

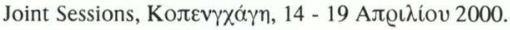

MAIR, P. - SMITH, G. (1990), Understanding Party System Change in Western

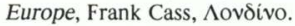

MCCOMBS, M.E. - SHAW, D. (1972), «The Agenda-Setting Function of the Mass

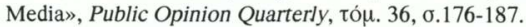

MIller, W. (1990), How Voters Change: The 1987 General Election Cam-

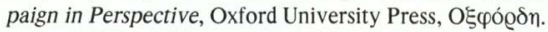

NORRIS, P. (2000), A Virtuous Circle? Political Communications in Post-Indus-

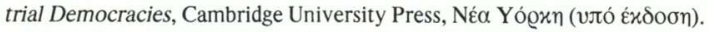
NORRIS, P. x.ó. (1999), On Message. Communicating the Campaign, Sage,

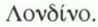

PESMAZOGLOU, S. (1993), «The 1980s in the Looking-Glass: PASOK and the

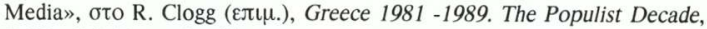

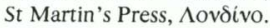

SWanson, D. - MANCini, P. (1996), Politics, Media and Modern Democracy. 
An International Study of Campaign Innovations in Election Campaigning and their Consequences, Praeger, Westport, Conn.

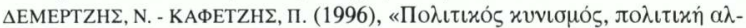

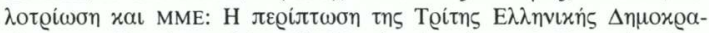

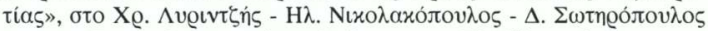

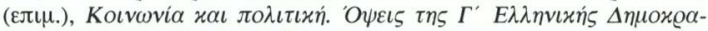

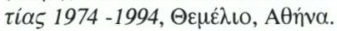

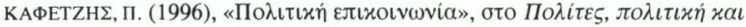

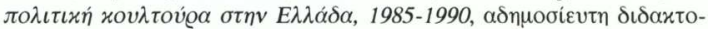

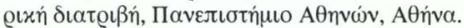

КАФЕTZH

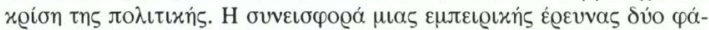

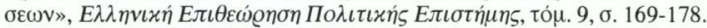

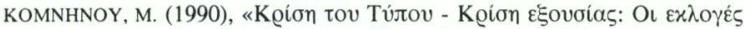

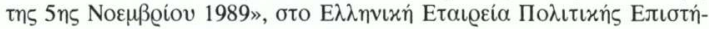

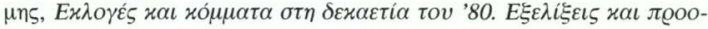

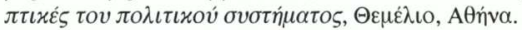

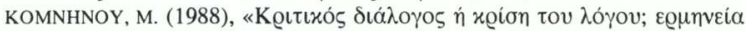

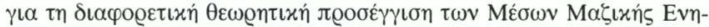

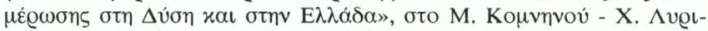

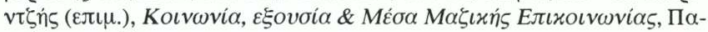
$\pi \alpha \zeta \dot{\eta} \sigma \eta 5$, A $\theta \dot{\eta} v \alpha$.

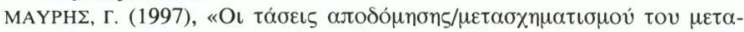

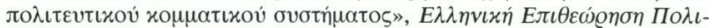

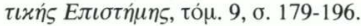

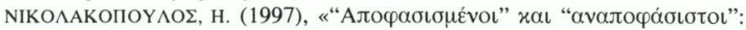

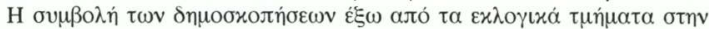

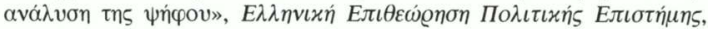
тó $\mu$. 9, б. 197-207.

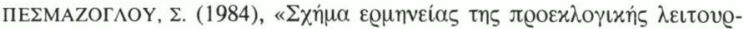

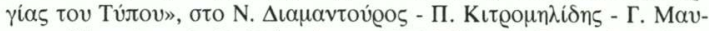

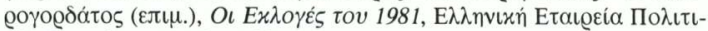

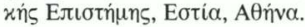

\section{HAEKTPONIKE $\triangle I E Y \Theta Y N \Sigma E I \Sigma$}

http://www.essex.ac.uk/ecpr/jointsessions/Copenhagen/papers/ws3/mcallister. PDF

http://www.ksg.harvard.edu/people/pnorris/book2.htm 